\title{
EQUIVARIANT ELLIPTIC GENERA AND LOCAL MCKAY CORRESPONDENCES*
}

\author{
ROBERT WAELDER ${ }^{\dagger}$
}

\begin{abstract}
In this paper we prove an equivariant version of the McKay correspondence for the elliptic genus on open varieties with a torus action. As a consequence, we will prove the equivariant DMVV formula for the Hilbert scheme of points on $\mathbb{C}^{2}$.
\end{abstract}

Key words. Elliptic genus, equivariant cohomology, McKay, localization, Hilbert scheme

AMS subject classifications. Primary 14E99; Secondary 55N91

1. Introduction. Let $X$ be a smooth variety, and $G$ a finite group acting holomorphically on $X$. Suppose that the quotient $X / G$ possesses a crepant resolution of singularities $V$. The McKay correspondence refers to the identification of topological invariants of $V$ with orbifold analogues of these invariants associated to the action of $G$ on $X$. The classical example is the case in which $G \subset S U(2)$ is a finite subgroup acting on $\mathbb{C}^{2}$. Then the quotient $\mathbb{C}^{2} / G$ possesses a unique crepant resolution $V$, and the Euler characteristic of $V$ coincides with the orbifold Euler characteristic $e_{\text {orb }}\left(\mathbb{C}^{2}, G\right)=\frac{1}{|G|} \sum_{g h=h g} e\left(\left(\mathbb{C}^{2}\right)^{g, h}\right)$. Over the years, the McKay correspondence has exhibited a remarkable versatility towards generalization. In [5] Batyrev investigated a more general class of resolutions $\widetilde{X / G} \rightarrow X / G$, and proved the McKay correspondence for the $E$-function in this situation. More recently, Borisov and Libgober have proven a similar result for the elliptic genus [7].

In this paper we will prove an equivariant analogue of the McKay Correspondence for the elliptic genus. The advantage of working in the equivariant setting is that, by localization, we can make sense of the elliptic genus even for open varieties. This allows us to prove a host of new formulas. One consequence of the work in this paper is a beautiful formula for the generating function of the equivariant elliptic genus of the Hilbert scheme of points on $\mathbb{C}^{2}$ (with the standard torus action):

$$
\sum_{n>0} p^{n} \operatorname{Ell}\left(\left(\mathbb{C}^{2}\right)^{[n]} ; y, q, t_{1}, t_{2}\right)=\prod_{m \geq 0, n>0, \ell, k} \frac{1}{\left(1-p^{n} q^{m} y^{\ell} t_{1}^{k_{1}} t_{2}^{k_{2}}\right)^{c(n m, \ell, k)}} .
$$

The terms $c(m, \ell, k)$ are the coefficients in the expansion of $\operatorname{Ell}\left(\mathbb{C}^{2} ; y, q, t_{1}, t_{2}\right)$ in $y, q, t_{1}$, and $t_{2}$. The above formula is an equivariant generalization of the DMVV formula:

$$
\sum_{n>0} p^{n} \operatorname{Ell}\left(S^{[n]} ; y, q\right)=\prod_{m \geq 0, n>0, \ell, k} \frac{1}{\left(1-p^{n} q^{m} y^{\ell}\right)^{c(n m, \ell, k)}}
$$

In the above formula, $S$ is a compact algebraic surface, and $\operatorname{Ell}\left(S^{[n]} ; y, q\right)$ is the elliptic genus of the Hilbert scheme of $n$ points on $S$. The non-equivariant DMVV formula was conjectured by string theorists Dijkgraaf, Moore, Verlinde and Verlinde [9], and proven by Borisov and Libgober [7]. The equivariant version is a conjecture of $\mathrm{Li}$, Liu, and Zhou [15].

\footnotetext{
*Received March 10, 2007; accepted for publication March 20, 2008.

${ }^{\dagger}$ UCLA Deptartment of Mathematics, Box 951555, Los Angeles, CA 90095-1555, USA (rwaelder@ math.ucla.edu).
} 
1.1. Background on the Elliptic Genus. For $X$ a smooth complex manifold, the elliptic genus of $X$ is defined as:

$$
\operatorname{Ell}(X)=\int_{X} \prod \frac{x_{j} \theta\left(\frac{x_{j}}{2 \pi i}-z, \tau\right)}{\theta\left(\frac{x_{j}}{2 \pi i}, \tau\right)} .
$$

The product is taken over the Chern roots of the holomorphic tangent bundle to $X$. $\theta(\cdot, \tau)$ is the Jacobi theta function, and $z$ represents a formal parameter. Setting $y=e^{2 \pi i z}$ and $q=e^{2 \pi i \tau}$, the elliptic genus may also be interpreted as the index of the following differential operator:

$$
y^{-d / 2} \bar{\partial} \otimes \bigotimes_{n=1}^{\infty} \Lambda_{-y q^{n-1}} T^{*} X \otimes \Lambda_{-y^{-1} q^{n}} T X \otimes S_{q^{n}} T^{*} X \otimes S_{q^{n}} T X .
$$

The modular properties of the Jacobi theta function endow the elliptic genus with a rich amount of structure. For example, if $X$ is Calabi-Yau, then the elliptic genus is a weak Jacobi form as a function of $(z, \tau) \in \mathbb{C} \times \mathbb{H}$. If $X$ is Calabi-Yau and possesses a nontrivial torus action, Liu has shown that the modular properties of the equivariant index of 2 actually imply its rigidity [16]. In addition to these properties, the elliptic genus encodes a large number of classical algebraic and topological invariants of the space. For example, letting $q \rightarrow 0$ in the expression for the elliptic genus produces $y^{-d / 2}$ times the Hirzebruch $\chi_{-y}$ genus, whereas letting $y \rightarrow 1$ produces the Euler characteristic of the space.

In [17], [6], Chin-Lung Wang, Borisov, and Libgober investigated the following relative version of the elliptic genus for pairs $(X, D)$, where $D=\sum_{i} a_{i} D_{i}$ is a smooth divisor with normal crossings and coefficients $a_{i} \neq 1$ :

$$
\operatorname{Ell}(X, D)=\int_{X} \prod \frac{x_{j} \theta\left(\frac{x_{j}}{2 \pi i}-z\right) \frac{\theta^{\prime}(0)}{2 \pi i}}{\theta\left(\frac{x_{j}}{2 \pi i}\right) \theta(-z)} \prod_{i} \frac{\theta\left(\frac{c_{1}\left(D_{i}\right)}{2 \pi i}-\left(-a_{i}+1\right) z\right) \theta(z)}{\theta\left(\frac{c_{1}\left(D_{i}\right)}{2 \pi i}-z\right) \theta\left(\left(-a_{i}+1\right) z\right)} .
$$

The modular properties of the Jacobi theta function imply that the relative elliptic genus satisfies the following change of variables formula for blow-up morphisms: If $f: \widetilde{X} \rightarrow X$ is the blow-up of $X$ along a smooth base with normal crossings with respect to the components of $D$, and $\widetilde{D}$ is the divisor on $\widetilde{X}$ satisfying: $K_{\widetilde{X}}+\widetilde{D}=$ $f^{*}\left(K_{X}+D\right)$, then $\operatorname{Ell}(\widetilde{X}, \widetilde{D})=\operatorname{Ell}(X, D)$. For $Z$ a $\mathbb{Q}$-Gorenstein variety with logterminal singularities, and $X \rightarrow Z$ a resolution of singularities with exceptional locus a normal crossing divisor $D$, Borisov and Libgober define the singular elliptic genus of $Z$ to be the relative elliptic genus of $(X, D)$. The change of variable formula, together with the Weak Factorization Theorem [1] implies that this definition is well-defined. Moreover, when $Z$ possesses a crepant resolution $V$, the singular elliptic genus of $Z$ is easily seen to coincide with the elliptic genus of $V$.

The singular elliptic genus (and its orbifold analogue) plays a crucial role in Borisov and Libgober's proof of the McKay correspondence for elliptic genera. Its utility stems from the fact that it behaves well with respect to a large class of birational modifications. The added flexibility obtained from studying the singular elliptic genus allowed Borisov and Libgober to reduce their proof to calculations involving toroidal embeddings. Their approach is similar in spirit to that of Batyrev in [5], who proved the McKay correspondence for the $E$-function by using the change of variables formula from motivic integration to reduce the case to calculations on toric varieties.

When $X$ has a nontrivial torus action, we may define the equivariant elliptic genus of $X$ to be the equivariant index of the operator defined in 2. By the index 
theorem [4], this is the same as the integral in equation 1 obtained by replacing the Chern roots of $T X$ with their equivariant analogues. Similarly, we may define an equivariant version of the relative elliptic genus by replacing appearances of $c_{1}\left(D_{i}\right)$ with their equivariant extensions.

The bulk of this paper is devoted to proving the change of variables formula for the equivariant orbifold elliptic genus (this case subsumes the non-orbifold case). Once we establish the change of variables formula in this situation, the remaining steps in the proof of the equivariant McKay correspondence for the elliptic genus follow closely the steps given in [7].

1.2. Outline of the Proof. In a recent preprint [18], I proved the equivariant change of variable formula for blow-ups along complete intersections $W=D_{1} \cap \ldots \cap$ $D_{k} \subset X$. The idea was to interpret the blow-down $\widetilde{X} \rightarrow X$ as a toroidal morphism. The stratification defined by the divisors $D_{i}$ determined the toroidal structure of $X$, whereas the stratification defined by the proper transforms of these divisors, together with the exceptional divisor, determined the toroidal structure of $\widetilde{X}$. The comparison of the relative elliptic genera of the base space and its blow-up was ultimately reduced to a computation involving the combinatorics of the polyhedral complexes associated to the two toroidal embeddings. This idea was inspired by Borisov and Libgober's use of polyhedral complexes in [7] to compute the push-forward of the orbifold elliptic class under the global quotient map.

Later it became apparant that the proof given in [18] could be adapted to the case in which $X$ was a "normal cone space", i.e., a fiber product of spaces $\mathbb{P}(F \oplus 1)$, where $F \rightarrow W$ was a holomorphic vectorbundle. The idea was that the Chern roots of the tautological quotient bundle $Q_{F} \rightarrow X$ should play the role of the "divisors" in a polyhedral complex associated to $X$. Similarly, if $f: \widetilde{X} \rightarrow X$ was the blow-up of $X$ along $W$ with exceptional divisor $E$, then the Chern roots of $f^{*} Q_{F} \otimes \mathcal{O}(-E)$, and of $\mathcal{O}(E)$ should behave like the "divisors" of a polyhedral complex associated to $\tilde{X}$. In this paper, we refer to such polyhedral complexes associated to the data of Chern roots as "twisted polyhedral complexes." The case of a general blow-up may be reduced to cases of this nature by using an equivariant version of deformation to the normal cone.

The breakdown of the sections in this paper are as follows: For generic specializations of the parameters $(z, \tau)$, the integrand of the equivariant elliptic genus is a power series in the equivariant parameters with differential form coefficients. In section 2 we discuss convergence issues related to power series of this type and put their corresponding cohomology theory on solid ground. In section 3 we define our principle objects of study; namely the equivariant orbifold elliptic class and its relative version. In sections 4 and 5 we review some facts about the equivariant cohomology of toric varieties and discuss how it relates to computing equivariant push-forwards of toroidal morphisms. In section 6 we use these results to compute the pushforward of the orbifold elliptic class under a toroidal morphism which is birational to a quotient by a finite group. This result is the equivariant analogue of Lemma 5.4 in [7]. Sections 7 to 10 are devoted to the proof of the equivariant change of variable formula. In section 7 , we prove an equivariant analogue of deformation to the normal cone, tailored specifically to handle cohomological data like the orbifold elliptic class. As stated above, this will allow us to reduce the proof of the change of variable formula to the case when $X$ is a normal cone space. In section 8 we prove for completeness a number of technical lemmas regarding spaces of this form. In section 9 we introduce the twisted polyhedral complex for normal cone spaces. In 10 we apply the techniques 
from the preceding sections to prove the equivariant change of variables formula. Finally, in 11 we prove the equivariant McKay correspondence for elliptic genera, and the equivariant DMVV formula.

Acknowledgements. I wish to thank my advisor Professor Kefeng Liu for introducing me to elliptic genera and for his constant support, as well as Professor Anatoly Libgober for his feedback and help with technical aspects of his work.

\section{Equivariant Cohomology and Power Series.}

2.1. Preliminaries on Equivariant Cohomology. We begin by reviewing some basic aspects of equivariant cohomology. For a thorough reference on the subject see [3].

Let $M$ be a smooth manifold and $T$ a torus acting smoothly on $M$. Let $e_{1}, \ldots, e_{\ell}$ form a basis for the Lie algebra of $T$ which is dual to the linear forms $u_{1}, \ldots, u_{\ell}$. Every $X \in \mathfrak{t}$ defines a vectorfield $X$ on $M$ by the formula $X(p)=\left.\frac{d}{d t}\right|_{t=0} \exp (t X) \cdot p$. Define $\Omega_{T}^{*}(M)$ to be the ring of differential forms on $M$ which are annihilated by $\mathcal{L}_{X}$ for every $X \in \mathfrak{t}$. If we let $d_{\mathfrak{t}}=d+\sum_{\alpha=1}^{\ell} u_{\alpha} i_{e_{\alpha}}$, then $d_{\mathfrak{t}}$ defines an operator on $\Omega_{T}^{*}(M) \otimes \mathbb{C}\left[u_{1}, \ldots, u_{\ell}\right]$ and satisfies $d_{\mathfrak{t}}^{2}=0$. The Cartan model for equivariant cohomology is defined to be:

$$
H_{T}^{*}(M)_{\text {Cartan }}=\frac{\operatorname{ker} d_{\mathfrak{t}}}{\operatorname{imd} d_{\mathfrak{t}}} .
$$

Alternatively, we could define the equivariant cohomology of $M$ via the following approach known as the Borel model: Let $E T=\left(S^{\infty}\right)^{\ell}$. ET is a contractible space on which $T$ acts freely. The diagonal action of $T$ on $M \times E T$ therefore gives rise to a smooth (infinite-dimensional) quotient $M_{T}=(M \times E T) / T$. It is easy to see that $M_{T}$ is a fiber bundle over $B T=E T / T$ with fiber $M$. Define the equivariant cohomology group $H_{T}^{*}(M)=H^{*}\left(M_{T}\right)$. As shown in [3], the Borel model for equivariant cohomology is isomorphic to the Cartan model defined above. One advantage to the Borel model is that it provides a convenient way to define equivariant characteristic classes. Namely, if $E$ is a $T$-vectorbundle over $M$ of rank $r$, then $E_{T}$ is a rank $r$ vectorbundle over $M_{T}$, and we may define the equivariant characteristic classes of $E$ to be the characteristic classes of $E_{T}$ which take values in $H^{*}\left(M_{T}\right) \cong H_{T}^{*}(M)$.

The Borel model point of view also provides a convenient way to extend the concept of Chern roots to the equivariant category. We first recall the definition of Chern roots. If $E$ is a complex vectorbundle over $M$, the flag bundle $\pi: \operatorname{Fl}(E) \rightarrow M$ is the fiber bundle whose fiber over a point $p$ is the space of flags $\Lambda_{1} \subset \Lambda_{2} \ldots \subset \Lambda_{r} \cong E_{p}$ with $\operatorname{dim} \Lambda_{i} / \Lambda_{i-1}=1$. The pullback bundle $\pi^{*} E$ splits into a direct some of line bundles $L_{i}$, and we define the Chern roots $x_{i}$ of $\pi^{*} E$ to be the first Chern classes of these line bundles $L_{i}$. The functorial nature of Chern classes implies that the elementary symmetric functions in $x_{i}$ are simply the pullbacks via $\pi^{*}$ of the Chern classes of $E$. Since $\pi^{*}$ is injective, this gives us a one to one correspondence between formulas involving the Chern classes of $E$ and formulas involving the Chern roots of $E$ which are symmetric with respect to permutations of the variables $x_{i}$. Analogously, if $E$ is a $T$-vectorbundle, we may define $\pi: \operatorname{Fl}\left(E_{T}\right) \rightarrow M_{T}$ as before. The pullback bundle $\pi^{*} E_{T}$ again splits into a sum of line bundles $L_{i}$, and we define the equivariant Chern roots to be the first Chern classes of these line bundles.

The translation of other concepts from cohomology to equivariant cohomology is more or less routine. For example, in either the Cartan or Borel model, one easily sees that a $T$-map $f: M \rightarrow N$ induces a pullback $f^{*}: H_{T}^{*}(N) \rightarrow H_{T}^{*}(M)$ as in 
ordinary cohomology. If $f: M \rightarrow N$ is a proper map of $T$-spaces, we also have an equivariant analogue of the cohomological push-forward $f_{*}: H_{T}^{*}(M) \rightarrow H_{T}^{*}(N)$. As in the non-equivariant setting, $f_{*}$ is functorial with respect to compositions of $T$-maps, and satisfies the projection formula $f_{*}\left(f^{*}(\omega) \wedge \eta\right)=\omega \wedge f_{*} \eta$. A new feature in equivariant cohomology is that we have an explicit expression for the restriction of $f_{*} \omega$ to a $T$-fixed component in $N$. This is given by the localization formula, which we discuss below.

If $p$ is a single point with trivial $T$-action, the equivariant map $\pi: M \rightarrow p$ induces a map $\pi^{*}: H_{T}^{*}(p) \rightarrow H_{T}^{*}(M)$. Since $H_{T}^{*}(p)=\mathbb{C}\left[u_{1}, \ldots, u_{\ell}\right]$, the map $\pi^{*}$ makes $H_{T}^{*}(M)$ into a $\mathbb{C}\left[u_{1}, \ldots, u_{\ell}\right]$-module. Define $H_{T}^{*}(M)_{l o c}=H_{T}^{*}(X) \otimes_{\mathbb{C}\left[u_{1}, \ldots, u_{\ell}\right]} \mathbb{C}\left(u_{1}, \ldots, u_{\ell}\right)$. A fundamental result of the subject is the localization theorem:

THEOREM 1. Let $\{P\}$ denote the set of $T$-fixed components of $M$. Then $H_{T}^{*}(M)_{l o c} \cong \bigoplus_{P} H^{*}(P) \otimes \mathbb{C}\left(u_{1}, \ldots, u_{\ell}\right)$.

If $P$ is a fixed component of $M$, the normal bundle to $P$ splits as a sum over the characters of the $T$-action on the fibers: $N_{P}=\bigoplus_{\lambda} V_{\lambda}$. Let $n_{\lambda}^{i}$ denote the formal Chern roots of $V_{\lambda}$. If we identify the equivariant parameters $u_{1}, \ldots, u_{\ell}$ with linear forms on the Lie algebra of $T$, then the equivariant Euler class $e(P)$ of $N_{P}$ is equal to $\prod_{\lambda} \prod_{i}\left(n_{\lambda}^{i}+\lambda\right)$. Since none of the characters $\lambda$ are equal to zero, we see that $e(P)$ is always invertible. In light of this fact, we can describe the above isomorphism more explicitly. The map $H_{T}^{*}(M)_{l o c} \rightarrow \bigoplus_{P} H^{*}(P) \otimes \mathbb{C}\left(u_{1}, \ldots, u_{\ell}\right)$ is given by $\omega \mapsto$ $\bigoplus_{P} \frac{i_{P}^{*} \omega}{e(P)}$, where $i_{P}: P \hookrightarrow M$ is the inclusion map.

Now if $f: M \rightarrow N$ is a proper map of $T$-spaces, we can compute $f_{*}$ in $H^{*}(N)_{l o c}$ using the functorial localization formula [13] [14]:

Theorem 2. Let $f: M \rightarrow N$ be a proper map of $T$-spaces. Let $P$ be a fixed component of $N$ and let $\{F\}$ be the collection of fixed components in $M$ which $f$ maps into P. Let $\omega \in H_{T}^{*}(M)$. Then:

$$
\sum_{F} f_{*} \frac{i_{F}^{*} \omega}{e(F)}=\frac{i_{P}^{*} f_{*} \omega}{e(P)}
$$

Throughout this paper we make frequent use of the equivariant Thom class of a vectorbundle. Recall that if $E$ is a vectorbundle over $M$, the Thom class $[\Phi] \in$ $H_{c v}^{*}(E)$ is a cohomology class with degree equal to the rank of $E$ which has compact support in the direction of the fibers of $E$ and which integrates to one over each fiber. (The notation $H_{c v}^{*}(\cdot)$ means compact support in the vertical direction.) If $E$ is a $T$ vectorbundle over a $T$-manifold $M$, by averaging over $T$, we can always assume that a representative of $[\Phi]$ is $T$-invariant. We can then uniquely extend the Thom class of $E$ to an equivariant class in the following sense:

Proposition 1. Let $E$ be a T-vectorbundle over a T-manifold $M$, and let $\Phi$ be a $T$-invariant representative of the Thom class. Then we can find a lower degree form $\eta \in \Omega_{T}^{*}(M) \otimes \mathbb{C}\left[u_{1}, \ldots, u_{\ell}\right]$ with compact support in the fiber direction so that $\Phi+\eta$ is $d_{\mathfrak{t}}$-closed. We call $\Phi+\eta$ the equivariant extension of $\Phi$. Any two such equivariant extensions of $\Phi$ differ by a $d_{\mathfrak{t}}$-exact form.

Proof. We assume for convenience that $T=S^{1}$. The general argument is the same but notationally more cumbersome. Thus $d_{\mathfrak{t}}=d+u i_{X}$, where $X$ is the vectorfield generated by the $S^{1}$ action. Let $\omega_{1}=i_{X} \Phi$. Then $\omega_{1}$ is $T$-invariant and $d \omega_{1}=$ $d i_{X} \Phi=0$ since $\Phi$ is closed and $T$-invariant. Since $\omega_{1}$ has smaller degree than $\Phi$ and 
compact support in the fiber direction, by the Thom isomorpism theorem, $\omega_{1}=d \eta_{1}$ for some form $\eta_{1}$ with compact support in the fiber direction. By averaging over $T$, we may assume $\eta_{1}$ is $T$-invariant. Note now that $\left(d+u i_{X}\right)\left(\Phi-u \eta_{1}\right)=-u^{2} i_{X} \eta_{1}$. If we let $\omega_{2}=i_{X} \eta_{1}$, then $d \omega_{2}=-i_{X} d \eta_{1}=-i_{X} \circ i_{X} \Phi=0$. Therefore, by the Thom isomorphism theorem again, $\omega_{2}=d \eta_{2}$, where $\eta_{2}$ is a $T$-invariant form with compact support in the fiber direction. Now $\left(d+u i_{X}\right)\left(\Phi-u \eta_{1}+u^{2} \eta_{2}\right)=u^{3} i_{X} \eta_{3}$. In general, then, if we define $\omega_{j}=i_{X} \eta_{j-1}$ and $\eta_{j}$ to be a $T$-invariant form with compact support in the fiber direction satisfying $d \eta_{j}=\omega_{j}$, then $\Phi+\sum_{j}(-1)^{j} u^{j} \eta_{j}$ is $d+u i_{X}$-closed, and is therefore an equivariant extension of $\Phi$.

Now suppose that $\eta$ is any equivariant cohomology class with top degree $\leq \operatorname{rk}(E)$ which has has compact support in the fiber direction and integrates to zero over each fiber. We will show that $\eta$ is $d_{\mathfrak{t}}$-exact. This clearly shows that any two equivariant extensions of the Thom class differ by a $d_{\mathfrak{t}}$-exact form. We may write the top degree $d$ part of $\eta$ as $\eta^{[d]}=\sum \eta_{i_{1}, \ldots, i_{\ell}}^{[d]} u_{1}^{i_{1}} \cdots u_{\ell}^{i_{\ell}}$, where $\eta_{i_{1}, \ldots, i_{\ell}}^{[d]}$ are $T$-invariant with compact support in the fiber direction. Since $d \eta^{[d]}=0$, we must have $d \eta_{i_{1}, \ldots, i_{\ell}}^{[d]}=0$. By the Thom isomorphism theorem, $\eta_{i_{1}, \ldots, i_{\ell}}^{[d]}=d \omega_{i_{1}, \ldots, i_{\ell}}$, where $\omega_{i_{1}, \ldots, i_{\ell}}$ is compactly supported in the fiber direction. By averaging over $T$ we may also assume that the $\omega$ 's are $T$-invariant. Therefore, subtract off $d_{\mathfrak{t}} \sum \omega_{i_{1}, \ldots, i_{\ell}} u_{1}^{i_{1}} \cdots u_{\ell}^{i_{\ell}}$ from $\eta$ and proceed by induction on the top degree of $\eta$.

Note that the proof of the above proposition implies the equivariant Thom isomorphism theorem:

THEOREM 3. Let $E$ be a T-vectorbundle over a compact T-manifold $M$, and $\pi: E \rightarrow M$ the projection from $E$ to $M$. Then $H_{T}^{*}(M)$ is isomorphic to space of equivariant cohomology classes in $E$ with compact support. The isomorphism is given by pulling back a class from $M$ and multiplying by the equivariant Thom class.

Proof. Let $\omega \in H_{T}^{*}(M)$, and let $\Phi$ be a representative of the equivariant Thom class of $E$. By the projection formula, which holds on the level of forms, $\pi_{*}\left(\pi^{*} \omega \wedge \Phi\right)=$ $\omega$, so the map $\pi^{*}(\cdot) \wedge[\Phi]$ is injective. Conversely, for $\alpha$ a representative of a compactly supported equivariant cohomology class on $E$, let $\pi_{*} \alpha$ denote the integral of $\alpha$ over the fibers of $E$. Then $\pi_{*} \alpha$ is an equivariantly closed form on $M$. Moreover, $\alpha-\pi^{*}\left(\pi_{*} \alpha\right) \wedge \Phi$ clearly integrates to zero over every fiber of $E$. Thus the proof of the above proposition implies $\alpha$ is cohomologous to $\pi^{*}\left(\pi_{*} \alpha\right) \wedge \Phi$.

2.2. Power Series in Equivariant Cohomology. For simplicity, we assume that $M$ has a $T=S^{1}$ action. Let $X$ be the vectorfield on $M$ induced by the action of $T$. Let $C(M)$ denote the ring of formal power series in $u$ with coefficients in $\Omega^{*}(M)^{T} \otimes \mathbb{C}$. Then $d_{X}=d-u i_{X}: C(M) \rightarrow C(M)$. We define $H^{*}(C(M))=\operatorname{ker} d_{X} /$ im $d_{X}$.

Multiplication by $u$ gives $H^{*}(C(M))$ the structure of a $\mathbb{C}[u]$-module. Given any $\mathbb{C}[u]$-module $A$, we define $A_{l o c}=A \otimes \mathbb{C}(u)$. Here, we interpret rational polynomials of the form $\frac{1}{1+u f(u)}$ as convergent power series by expanding around $u=0$.

Let $g$ be a $T$-invariant metric on $M$, and define $\theta=g(X, \cdot)$. Let $F \subset M$ be the $T$-fixed locus, and let $\rho$ be a bump function identically equal to one outside a tubular neighborhood of $F$, and equal to zero inside a smaller tubular neighborhood. (Note that we allow $F$ to have different components of varying dimensions). Then $\rho\left(d_{X} \theta\right)^{-1}$ is a well-defined element of $C(M)_{l o c}$. Furthermore, it is easy to see that for any closed form $\omega \in C(M)_{l o c}, \omega-d_{X}\left(\omega \cdot \theta \cdot \rho\left(d_{X} \theta\right)^{-1}\right)$ has compact support in a tubular neighborhood of $F$. It follows that every closed form in $C(M)_{l o c}$ may be represented by a form with compact support in a tubular neighborhood of $F$. Let 
$C\left(\nu_{F}\right)_{c}$ denote the ring of formal power series in $u$ whose coefficients are $T$-invariant forms with compact support in the normal bundle $\nu_{F}$.

Let $\pi_{*}: C\left(\nu_{F}\right)_{c, l o c} \rightarrow C(F)_{l o c}$ be the map $\sum \omega_{n} u^{n} \mapsto \sum \pi_{*} \omega_{n} u^{n}$, where $\pi_{*} \omega_{n}$ denotes the integral of $\omega_{n}$ over the fiber of $\nu_{F}$. We first remark that $\pi_{*} i_{X} \omega=0$ for any differential form $\omega$ with compact support in $\nu_{F}$. To see this, note that we can always express $\omega$ locally in the form $\omega=f(x, t) \pi^{*} \phi d t_{1} \ldots d t_{k}$. Here $x$ represents the coordinates along $F, t$ represents the coordinates along the fiber, and $\pi^{*} \phi$ is the pullback of a form on $F$ via the projection $\pi: \nu_{F} \rightarrow F$. Thus $i_{X} \omega=f(x, t) \pi^{*} \phi$. $i_{X} d t_{1} \ldots d t_{k}$ which clearly integrates to zero along the fiber, since the degree of the form along the fiber is necessarily smaller than the fiber dimension.

It follows that $\pi_{*} d_{X}=d \pi_{*}$, and therefore it induces a map in cohomology $\pi_{*}$ : $H^{*}\left(C\left(\nu_{F}\right)_{c}\right)_{l o c} \rightarrow H^{*}(C(F))_{l o c}$.

Theorem 4. $\pi_{*}$ is injective.

Proof. Let $\omega=\sum_{n} \omega_{n} u^{n} \in C\left(\nu_{F}\right)_{c, l o c}$ be closed. Suppose $\pi_{*} \omega=d \eta$. By subtracting off $\pi^{*} d \eta \cdot \Phi$, where $\Phi$ is the equivariant Thom class of $\nu_{F}$, we may reduce to the case in which $\pi_{*} \omega=0$. For any differential form $\alpha$, denote by $\alpha[i]$ the degree $i$ part. Then for some $0 \leq k \leq \operatorname{dim} M, \omega=\omega[k]+\omega[k-1]+\ldots+\omega[0]$, where $\omega[i]=\sum \omega_{n}[i] u^{n}$. Since $\omega$ is $d_{X}$-closed, $d \omega_{n}[k]=0$. Since $\pi_{*} \omega[k]=\sum \pi_{*} \omega_{n}[k] u^{n}=0$, by the Thom isomorphism theorem, we can find compactly supported forms $y_{n}$ such that $d y_{n}=\omega_{n}[k]$. By averaging over $T$, we may assume that these forms are $T$ invariant. Then $\omega-d_{X} \sum y_{n} u^{n-1}$ has top degree $<k$ and is annihilated by $\pi_{*}$. The proof then follows by induction. $\square$

Since $[\omega]=0$ if and only if $\pi_{*}[\omega]=0$, we see that $[\omega]=\left[\pi^{*} \pi_{*} \omega \cdot \Phi\right]$. Hence $\left.[\omega]\right|_{F}=\left[\pi_{*} \omega \cdot e\left(\nu_{F}\right)\right]$, where $e\left(\nu_{F}\right)$ is the equivariant Euler class. Since the Euler class is invertible, the restriction map $\left.[\omega] \mapsto[\omega]\right|_{F}$ must be invertible. This proves the localization theorem for $H^{*}(C(M))_{l o c}$ :

Theorem 5. Let $F \subset M$ denote the fixed locus of $T$. Then the restriction map $H^{*}(C(M))_{l o c} \rightarrow H^{*}(C(F))_{l o c}$ is an isomorphism.

We now introduce the ring of analytic forms: Let $C^{a n}(M) \subset C(M)$ denote the ring of forms $\sum \omega_{n} u^{n}$ with the property that the partial sums $\sum_{n=0}^{N} \omega_{n} s^{n}$ converge in the $C^{\infty}$ sense to a form $\omega \in \Omega^{*}(M)^{T}$ for $\|s\|$ sufficiently small (meaning that the sums converge uniformly on compact sets, as well as all derivatives of these sums with respect to coordinate differentiation). Let $B^{a n}(M)=d_{X}(C(M)) \cap C^{a n}(M)$. We define

$$
H^{*}\left(C^{a n}(M)\right)=\frac{\operatorname{ker} d_{X}: C^{a n}(M) \rightarrow C^{a n}(M)}{B^{a n}(M)} .
$$

If $F$ denotes the $T$-fixed locus of $M$, the above proof of the localization theorem extends word for word to the case of analytic forms.

For $s \in \mathbb{C}^{*}$, following Witten [19], let $d_{s}=d-s i_{X}: \Omega^{*}(M)^{T} \rightarrow \Omega^{*}(M)^{T}$. Define $H_{s}^{*}(M)=\frac{\operatorname{ker} d_{s}}{\operatorname{im} d_{s}}$. Again, the above proof of the localization theorem adapts easily to the case of $H_{s}^{*}(M)$. Define an equivalence relation $\sim$ on $\prod_{s \in \mathbb{C}^{*}} H_{s}^{*}(M)$ as follows: For $\omega, \omega^{\prime} \in \prod_{s \in \mathbb{C}^{*}} H_{s}^{*}(M)$, we say that $\omega \sim \omega^{\prime}$ if $\omega_{s}=\omega_{s}^{\prime}$ for $\|s\|$ sufficiently small. By $\omega_{s}$, of course, we mean the $s$-component of $\omega$. We denote the group $\prod_{s \in \mathbb{C}^{*}} H_{s}^{*}(M) / \sim$ by $W$.

Proposition 2. There is a natural evaluation map ev $: H^{*}\left(C^{a n}(M)\right) \rightarrow W$ given by $\left[\sum \omega_{n} u^{n}\right] \rightarrow\left[\sum \omega_{n} s^{n}\right]$. 
Proof. Let $\omega=\sum \omega_{n} u^{n} \in C^{a n}(M)$ be a closed form, and let $d_{X} \sum \eta_{n} u^{n} \in$ $C^{a n}(M)$. We wish to show that for $\|s\|$ sufficiently small, $\left[\sum \omega_{n} s^{n}\right]=\left[\sum \omega_{n} s^{n}+\right.$ $\left.d_{s} \sum \eta_{n} s^{n}\right]$ in $H_{s}^{*}(M)$. First, for $\|s\|$ sufficiently small, both sums $\sum \omega_{n} s^{n}$ and $\sum d_{s} \eta_{n} s^{n}$ converge. Therefore, it suffices to prove that $\sum d_{s} \eta_{n} s^{n}$ is exact for these values of $s$. Let $F \subset M$ be the $T$-fixed locus. Then $\left.\sum d_{s} \eta_{n}\right|_{F} s^{n}=\left.\sum d \eta_{n}\right|_{F} s^{n}$. If we let $\Delta$ denote the Hodge Laplacian, then by Hodge theory, a form is exact if and only if it is orthogonal to the kernel of $\Delta$. Now every partial sum of $\left.\sum d \eta_{n}\right|_{F} s^{n}$ is exact, and hence orthogonal to the kernel of $\Delta$. This orthogonality is clearly preserved when we take the limit as $n \rightarrow \infty$. Thus $\left.\sum d \eta_{n}\right|_{F} s^{n}$ converges to an exact form. Hence $\left.\left[\sum d_{s} \eta_{n} s^{n}\right]\right|_{F}=0$, and therefore $\left[\sum d_{s} \eta_{n} s^{n}\right]=0$ by localization.

Let $H\left(x_{1}, \ldots, x_{r}\right)$ be function which is holomorphic in a neighborhood of $(0, \ldots, 0)$. Let $H=\sum_{i_{1}, \ldots, i_{r}} a_{i_{1}, \ldots, i_{r}} x_{1}^{i_{1}} \cdots x_{r}^{i_{r}}$ be the corresponding power series expansion. Let $\left[\omega_{1}\right], \ldots,\left[\omega_{r}\right]$ be equivariant forms in $H_{T}^{*}(M)$ such that $u \mid \operatorname{deg}_{0} \omega_{i}$. Note that this property is independent of the choice of representatives for $\left[\omega_{i}\right]$. Then $H\left(\left[\omega_{1}\right], \ldots,\left[\omega_{r}\right]\right)$ is a well-defined element of $H^{*}\left(C^{a n}(M)\right)$. We can see this as follows:

Fix representatives $\omega_{1}, \ldots, \omega_{r} \in C^{a n}(M)$. Write $\omega_{i}=\tilde{\omega}_{i}+f_{i} u$, where $f_{i} \in$ $C^{\infty}(M) \otimes \mathbb{C}[u]$ and $\operatorname{deg} \tilde{\omega}_{i}>0$. Then

$$
\begin{aligned}
H\left(\omega_{1}, \ldots, \omega_{r}\right) & =\sum_{i_{1}, \ldots, i_{r}} a_{i_{1}, \ldots, i_{r}}\left(\tilde{\omega}_{1}+f_{1} u\right)^{i_{1}} \cdots\left(\tilde{\omega}_{r}+f_{r} u\right)^{i_{r}} \\
& =\sum_{j_{1}, \ldots, j_{r}} \tilde{\omega}^{j_{1}} \cdots \tilde{\omega}_{r}^{j_{r}} \sum_{i_{1}, \ldots, i_{r}} a_{i_{1}, \ldots, i_{r}}\left(\begin{array}{c}
i_{1} \\
j_{1}
\end{array}\right) \cdots\left(\begin{array}{c}
i_{r} \\
j_{r}
\end{array}\right)\left(f_{1} u\right)^{i_{1}-j_{1}} \cdots\left(f_{r} u\right)^{i_{r}-j_{r}} \\
& =\sum_{j_{1}, \ldots, j_{r}} \partial^{\left(j_{1}\right)} \cdots \partial^{\left(j_{r}\right)} H\left(f_{1} u, \ldots, f_{r} u\right) \tilde{\omega}_{1}^{j_{1}} \cdots \tilde{\omega}_{r}^{j_{r}} .
\end{aligned}
$$

Here $\partial^{(k)}=\frac{\partial^{k}}{k !}$. The sum over $j_{1}, \ldots, j_{r} \geq 0$ is finite because the forms $\tilde{\omega}_{i}$ are nilpotent. It is therefore clear that the above expression is a well-defined $d_{X}$-closed form in $C^{a n}(M)$. We next show that the corresponding class in $H^{*}\left(C^{a n}(M)\right)$ is independent of the choice of generators for $\left[\omega_{i}\right]$. Let $\widetilde{H}\left(s_{1}, t_{1}, \ldots, s_{r}, t_{r}\right)=H\left(s_{1}+t_{1}, \ldots, s_{r}+t_{r}\right)$. Then $\widetilde{H}=H\left(s_{1}, \ldots, s_{r}\right)+t_{1} \tilde{H}_{1}+\ldots+t_{r} \tilde{H}_{r}$. Thus, $H\left(\omega_{1}+d_{X} \eta_{1}, \ldots, \omega_{r}+d_{X} \eta_{r}\right)=$ $\widetilde{H}\left(\omega_{1}, d_{X} \eta_{1}, \ldots, \omega_{r}, d_{X} \eta_{r}\right)=H\left(\omega_{1}, \ldots, \omega_{r}\right)+d_{X}\left(\eta_{1} \tilde{H}_{1}+\ldots+\eta_{r} \tilde{H}_{r}\right)$, so $H\left(\left[\omega_{1}\right], \ldots,\left[\omega_{r}\right]\right)$ is well-defined independent of our choice of generators. Before proceeding further, we point out an important property possessed by the forms of this type.

Let $\widehat{C}(M) \subset C^{a n}(M)$ denote the subring $\{$ closed invariant forms $\otimes \mathbb{C}\{u\}\}$ where $\mathbb{C}\{u\}$ denotes the ring of power series in $u$ which converge in sufficiently small neighborhoods of the origin. Let $\widehat{H}(M) \subset H^{*}\left(C^{a n}(M)\right)_{l o c}$ denote the subspace of forms $[\omega]$ such that $\left.\omega\right|_{F} \in \widehat{C}(F)_{l o c}$ for some representative $\omega \in[\omega]$. Then if $\left[\omega_{1}\right], \ldots,\left[\omega_{r}\right] \in H_{T}^{*}(M)$, and $u \mid \operatorname{deg}_{0} \omega_{i}$, then $H\left(\left[\omega_{1}\right], \ldots,\left[\omega_{r}\right]\right) \in \widehat{H}(M)$. This is because $\left.\operatorname{deg}_{0} \omega_{i}\right|_{F} \in \mathbb{C}[u]$ and $\left.d \tilde{\omega}_{i}\right|_{F}=0$. Note also that functorial localization holds in this situation, and implies that, for $f: M \rightarrow N, f_{*} \widehat{H}(M) \subset \widehat{H}(N)$.

Lemma 1. ev $: \widehat{H}(M) \rightarrow W(M)$ is injective.

Proof. Let $[\omega] \in \widehat{H}(M)$, so that $\left.\omega\right|_{F}=\omega_{1} f_{1}+\ldots+\omega_{k} f_{k}$, where $\omega_{i}$ are closed forms on $F$ and $f_{i}$ are convergent Laurent series in $u$ in some small disk about the origin. If $[\omega] \neq 0$, then by localization, $\left.[\omega]\right|_{F} \neq 0$. Without loss of generality, we may assume that $\omega_{1}, \ldots, \omega_{k}$ are linearly independent as cohomology classes. We can always choose an arbitrarily small $s$ so that $f_{1}(s), \ldots, f_{k}(s)$ are not all zero. We then have that $\left.e v[\omega]_{s}\right|_{F}=f_{1}(s)\left[\omega_{1}\right]+\ldots+f_{k}(s)\left[\omega_{k}\right] \neq 0$. Hence $e v[\omega] \neq 0$ in $W(M)$. 
All the above machinary was put together to make the following argument: Let $f: M \rightarrow N$, and let $H=H\left(\left[\omega_{1}\right], \ldots,\left[\omega_{r}\right]\right)$ for $\left[\omega_{i}\right] \in H_{T}^{*}(M), u \mid \operatorname{deg}_{0} \omega_{i}$. Suppose $\theta_{n}(s)$ is a sequence of $d_{s}$-closed forms which converge in the $C^{\infty}$ sense to a representative of $(e v H)_{s}$ for $\|s\|$ sufficiently small. If we factor $f$ as an inclusion followed by a projection, then $f_{*}$ makes sense on the form level and $f_{*} \theta_{n}(s)$ converge to representatives of $\left(f_{*} e v H\right)_{s}$. Now suppose that $\left[f_{*} \theta_{n}(s)\right]=\left[b_{n}(s)\right]$ and that $b_{n}(s) \rightarrow b(s)$. Then $f_{*} \theta_{n}(s)=b_{n}(s)+d_{s} \eta_{n}(s)$, and since $f_{*} \theta_{n}(s)$ and $b_{n}(s)$ converge, we have that $[b(s)]=\left[f_{*} e v H\right]_{s}$. Now if $s \mapsto[b(s)]$ corresponds to a form evG for some $G\left(\left[\alpha_{1}\right], \ldots,\left[\alpha_{k}\right]\right),\left[\alpha_{i}\right] \in H_{T}^{*}(N)$, then $f_{*} e v H=e v f_{*} H=e v G$, and therefore $f_{*} H=G$. Thus, we can compute push-forwards of summations by applying the push-forward term-by-term, so long as the corresponding summation converges in the sense discussed in this section. This observation will be used implicitly throughout this paper; for example, it will allow us in sections 5 and 9 to reduce computations involving convergent power series to computations involving polynomials.

REMARK 1. In what follows we will be interested in the case where $T$ is a compact torus of arbitrary dimension. It is easy to see how to generalize the above machinary to this situation. The difficulty lies more in the notation than in any other aspect.

3. The Orbifold Elliptic Class. Let $X$ be a smooth projective variety with a holomorphic $T \times G$ action. Let $D=\sum_{I} \alpha_{i} D_{i}$ be a smooth $G$-normal crossing divisor with $\alpha_{i}<1$. The $G$-normal condition means that if $g \in \operatorname{stab}_{G}(x)$ and $x \in D_{i}$, then $g \cdot D_{i}=D_{i}$. Let $X_{\gamma}^{g, h}$ be a connected component of the fixed locus $X^{g, h}$ for some commuting pair $g, h \in G$. Then $N_{X_{\gamma}^{g, h} / X}$ splits into character sub-bundles $\bigoplus_{\lambda} N_{\lambda}$, where $g$ (resp. $h$ ) acts on $N_{\lambda}$ as multiplication by $e^{2 \pi i \lambda(g)}$ (resp. $\left.e^{2 \pi i \lambda(h)}\right)$. Let $I_{\gamma}^{g, h} \subset I$ index the set of divisors $D_{i}^{X}$ which contain $X_{\gamma}^{g, h}$. Since $D$ is $G$-normal, $g$ (resp. $h$ ) acts on $\left.\mathcal{O}\left(D_{i}\right)\right|_{X_{\gamma}^{g, h}}$ as multiplication by $e^{2 \pi i \lambda_{i}(g)}$ (resp. $e^{2 \pi i \lambda_{i}(h)}$ ) for every $i \in I_{\gamma}^{g, h}$. For $i \notin I_{\gamma}^{g, h}$, we define $\lambda_{i}(g)=\lambda_{i}(h)=0$.

Following [7], we define the orbifold elliptic class associated to the pair $\left(X_{\gamma}^{g, h}, D\right)$ as follows: $\mathcal{E} l l_{\text {orb }}\left(X_{\gamma}^{g, h}, D\right)=$

$$
\begin{aligned}
& \left(i_{X_{\gamma}^{g}, h}\right)_{*}\left\{\prod_{T X_{\gamma}^{g, h}} \frac{\frac{x_{i}}{2 \pi i} \theta\left(\frac{x_{i}}{2 \pi i}-z\right) \theta^{\prime}(0)}{\theta\left(\frac{x_{i}}{2 \pi i}\right) \theta(-z)} \times\right. \\
& \left.\prod_{\lambda, N_{\lambda}} \frac{\theta\left(\frac{x_{\lambda, c}}{2 \pi i}+\lambda(g)-\lambda(h) \tau-z\right) \frac{\theta^{\prime}(0)}{2 \pi i}}{\theta\left(\frac{x_{\lambda, c}}{2 \pi i}+\lambda(g)-\lambda(h) \tau\right) \theta(-z)} e^{2 \pi i \lambda(h) z}\right\} \times \\
& \prod_{I} \frac{\theta\left(\frac{D_{i}}{2 \pi i}+\lambda_{i}(g)-\lambda_{i}(h) \tau-\left(-\alpha_{i}+1\right) z\right) \theta(-z)}{\theta\left(\frac{D_{i}}{2 \pi i}+\lambda_{i}(g)-\lambda_{i}(h) \tau-z\right) \theta\left(-\left(-\alpha_{i}+1\right) z\right)} e^{-2 \pi i \lambda_{i}(h) \alpha_{i} z}
\end{aligned}
$$

Here $x_{i}$ denote the equivariant Chern roots of $T X_{\gamma}^{g, h}, x_{\lambda, c}$ denote the equivariant Chern roots of $N_{\lambda}$, and (abusing notation) $D_{i}$ denote the equivariant first Chern classes of the corresponding divisors.

Finally we define the orbifold elliptic class of $(X, D, G)$ as follows:

$$
\mathcal{E} l l_{\text {orb }}(X, D, G)=\frac{1}{|G|} \sum_{g h=h g, \gamma} \mathcal{E} l l_{\text {orb }}\left(X_{\gamma}^{g, h}, D\right) \text {. }
$$

When $G=1$, we will write $\mathcal{E} l l(X, D)$ instead of $\mathcal{E} l l_{\text {orb }}(X, D, G)$ and refer to this object as the equivariant elliptic class of the pair $(X, D)$. We view all such classes as an elements inside the ring $\widehat{H}(X)$. 
Let $\{F\}$ denote the collection of fixed components of $X$. For each $F$ let $e(F)$ denote the equivariant Euler class of the normal bundle to $F$. We define the equivariant orbifold elliptic index

$$
E l l_{\text {orb }}(X, D, G) \equiv\left(\frac{2 \pi i \theta(-z)}{\theta^{\prime}(0)}\right)^{\operatorname{dim} X} \sum_{F} \int_{F} \frac{\mathcal{E} l l_{\text {orb }}(X, D, G)}{e(F)} .
$$

It is a convergent power series in the equivariant parameters which depends implicitly upon the value of the complex parameter $z$ and on the lattice parameter $\tau$ used in the definition of the Jacobi theta function. We define the equivariant elliptic index $\operatorname{Ell}(X, D)$ similarly.

4. Toric Varieties and Equivariant Cohomology. For a good reference on toric varieties, see [11]. Let $X$ be a smooth complete toric variety of dimension $n$. We denote the fan of $X$ by $\Sigma_{X}$, the lattice of $X$ by $N_{X}$, and the big torus by $T_{X}$. Let $Y$ be a smooth complete toric variety which satisfies the following properties:

(1): $N_{X} \subset N_{Y}$ is a finite index sublattice.

(2): $\Sigma_{X}$ is a refinement of $\Sigma_{Y}$ obtained by adding finitely-many one dimensional rays.

There is an obvious map of fans $\nu: \Sigma_{X} \rightarrow \Sigma_{Y}$ which induces a smooth map $\mu: X \rightarrow Y$. We call a map induced by such a morphism of fans a toric morphism. It is easy to verify that $\mu: T_{X} \rightarrow T_{Y}$ is a covering map with covering group $N_{Y} / N_{X}$. Thus, we may regard $Y$ as a $T_{X}$-space. Our goal in this section is to obtain a convenient description of the equivariant pushforward $\mu_{*}: H_{T}^{*}(X) \rightarrow H_{T}^{*}(Y)$ in terms of the combinatorics of $\Sigma_{X}$ and $\Sigma_{Y}$. Here $T=T_{X}$.

We first note that fixed points $F$ of $X$ are in $1-1$ correspondence with $n$ dimensional cones $C_{F} \subset \Sigma_{X}$. Furthermore, the infinitesimal weights of the $T$-action on $N_{F}$ correspond to linear forms in $\operatorname{Hom}\left(N_{X}, \mathbb{Z}\right)$ which are dual to the generators of $C_{F}$ in $N_{X}$. With this identification in mind, for any $\omega \in H_{T}^{*}(X)$, the the collection of polynomials $\left\{\left.\omega\right|_{F}\right\}_{F \in \operatorname{Fix}(X)}$ defines a piecewise polynomial function on the fan $\Sigma_{X}$. Define $\mathbb{C}\left[\Sigma_{X}\right]$ to be the ring of all piecewise polynomial functions on the fan of $X$. It is well-known that the map $H_{T}^{*}(X) \rightarrow \mathbb{C}\left[\Sigma_{X}\right]$ described here is an isomorphism (see, for example, [8]):

Theorem $6 . H_{T}^{*}(X) \cong \mathbb{C}\left[\Sigma_{X}\right]$.

Via this identification, we define $\nu_{*}: \mathbb{C}\left[\Sigma_{X}\right] \rightarrow \mathbb{C}\left[\Sigma_{Y}\right]$ to be the map which makes the following diagram commute:

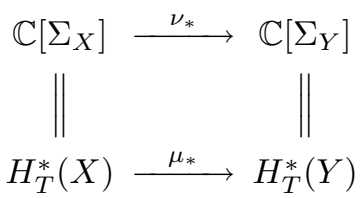

Here we understand $\mathbb{C}\left[\Sigma_{Y}\right]$ to be the ring of piecewise polynomial functions on $\Sigma_{Y}$ with respect to the lattice $N_{X}$.

We now describe $\nu_{*}$ more explicitly. First notice that for $f \in \mathbb{C}\left[\Sigma_{X}\right], \nu_{*} f$ is given by viewing $\left.f\right|_{F}$ as the zero degree part of an equivariant cohomology class $\omega \in H_{T}^{*}(X)$, pushing $\omega$ forward by $\mu_{*}$, and then forming the piecewise polynomial function defined by the zero degree part of $\mu_{*} \omega$. Thus, let $C \subset \Sigma_{Y}$ be an $n$-dimensional cone. Let $\nu^{-1} C$ be the fan $\Sigma_{C} \subset \Sigma_{X}$ which is the union of $n$-dimensional cones $C_{i}$. 
Let $x_{1}^{C_{i}}, \ldots, x_{n}^{C_{i}}$ be the linear forms dual to $C_{i}$ and $x_{1}^{C}, \ldots, x_{n}^{C}$ the linear forms in $\operatorname{Hom}\left(N_{Y}, \mathbb{Z}\right) \subset \operatorname{Hom}\left(N_{X}, \mathbb{Z}\right)$ dual to $C$. By functorial localization:

$$
\left(\nu_{*} f\right)_{C}=\sum_{C_{i} \subset \Sigma_{X}} f_{C_{i}} \frac{\prod_{j=1}^{n} x_{j}^{C}}{\prod_{j=1}^{n} x_{j}^{C_{i}}} .
$$

Similarly, we define $\nu^{*}: \mathbb{C}\left[\Sigma_{Y}\right] \rightarrow \mathbb{C}\left[\Sigma_{X}\right]$ to be the map which makes the following diagram commute:

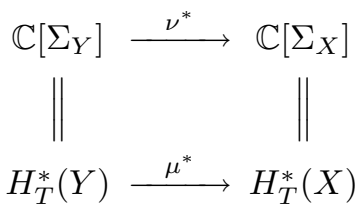

Proposition 3. $\nu^{*}(f)=f \circ \nu$

Proof. Let $\omega \in H_{T}^{*}(Y)$ be the form such that $\left.\omega\right|_{P}=\left.f\right|_{P}$ for every fixed point $P$. Let $F \in \mu^{-1}(P)$. Then

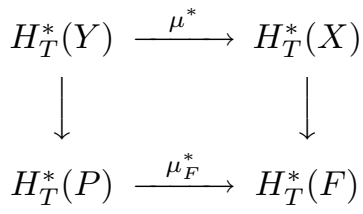

commutes. Hence $\left.\left(\mu^{*} \omega\right)\right|_{F}=\mu_{F}^{*}\left(\left.\omega\right|_{P}\right)=\mu_{F}^{*}\left(f_{P}\right)=f_{P}$. Thus $\nu^{*}(f)$ is the piecewise polynomial function which is equal to $f_{C_{P}}$ on every cone $C_{F} \in \nu^{-1} C_{P}$. This is precisely the piecewise polynomial $f \circ \nu$. $\square$

The map $\nu^{*}: \mathbb{C}\left[\Sigma_{Y}\right] \rightarrow \mathbb{C}\left[\Sigma_{X}\right]$ makes $\mathbb{C}\left[\Sigma_{X}\right]$ into a $\mathbb{C}\left[\Sigma_{Y}\right]$-module. As such, we observe:

Proposition 4. $\nu_{*}$ is a $\mathbb{C}\left[\Sigma_{Y}\right]$-module homomorphism.

Proof. In other words, we wish to prove the projection formula $\nu_{*}\left(f \nu^{*} g\right)=$ $\nu_{*}(f) \cdot g$. This follows from identifying $\nu_{*}$ with $\mu_{*}, \nu^{*}$ with $\mu^{*}$ and invoking the projection formula from equivariant cohomology. $\square$

\section{Toroidal Embeddings and Toroidal Morphisms.}

5.1. Definitions. Let $X$ be a compact variety and $D_{X}=\sum_{I_{X}} D_{i}^{X}$ a divisor on $X$ whose irreducible components are smooth normal crossing divisors. For $I \subset I_{X}$, let $X_{I, j}$ denote the $j$ th connected component of $\cap_{I} D_{i}^{X}$. Let $X_{I, j}^{o}=X_{I, j}-\cup_{I^{c}} D_{i}^{X}$. The collection of subvarieties $X_{I, j}^{o}$ form a stratification of $X$. Associated to these data is a polyhedral complex with integral structure defined as follows:

Corresponding to $X_{I, j}$, define $N_{I, j}=\mathbb{Z} e_{i_{1}, j}+\ldots+\mathbb{Z} e_{i_{k}, j}$ to be the free group on the elements $e_{i_{1}, j}, \ldots, e_{i_{k}, j}$. Here $i_{1}, \ldots i_{k}$ are the elements of $I$. Define $C_{I, j}$ to be the cone in the first orthant of this lattice. Whenever $I^{\prime} \subset I$ and $X_{I, j} \subset X_{I^{\prime}, j^{\prime}}$ we have natural inclusion maps $N_{I^{\prime}, j^{\prime}} \hookrightarrow N_{I, j}$ and $C_{I^{\prime}, j^{\prime}} \hookrightarrow C_{I, j}$. Define $\Sigma_{X}$ to be the polyhedral complex with integral structure obtained by gluing the cones $C_{I, j}$ together according to these inclusion maps.

Let $\mathbb{C}\left[\Sigma_{X}\right]$ denote the ring of piecewise polynomial functions on $\Sigma_{X}$. Fix $C \subset \Sigma_{X}$. Define $f^{C}$ to be the piecewise polynomial function which is equal to $\prod_{j=1}^{\operatorname{dim} C} x_{j}^{C}$ on 
every cone containing $C$, and equal to zero everywhere else. As in the toric geometry case, there is a natural correspondence between piecewise linear functions on $\Sigma_{X}$ and Cartier divisors whose irreducible components are components of $D_{X}$. We denote the piecewise linear function corresponding to $D$ by $f^{D}$.

5.2. Toroidal Morphisms. Our primary interest in this section is the study of toroidal morphisms. This is a map $\mu:\left(X, D_{X}, \Sigma_{X}\right) \rightarrow\left(Y, D_{Y}, \Sigma_{Y}\right)$ which satisfies the following:

(1): $\mu: X-D_{X} \rightarrow Y-D_{Y}$ is an unramified cover.

(2): $\mu$ maps the closure of a stratum in $X$ to the closure of a stratum in $Y$.

(3): Let $U_{y}$ be an analytic neighborhood of $y \in Y$ such that the components of $D_{Y}$ passing through $y$ correspond to coordinate hyperplanes. Then for $x \in \mu^{-1}(y)$, there exists an analytic neighborhood $U_{x}$ of $x$ such that the components of $D_{X}$ passing through $x$ correspond to coordinate hyperplanes of $U_{x}$. Moreover, the map $U_{x} \rightarrow U_{y}$ is given by monomial functions in the coordinates.

Corresponding to $\mu$, we can define a map $\nu: \Sigma_{X} \rightarrow \Sigma_{Y}$ as follows: Let $C_{I, i} \subset \Sigma_{X}$ and let $e_{1}, \ldots, e_{k} \in N_{I, i}$ be the generators of $C_{I, i}$ which correspond to the divisors $D_{1}^{X}, \ldots, D_{k}^{X}$. We have that $\mu\left(X_{I, i}\right)=Y_{J, j}$. Let $v_{1}, \ldots, v_{\ell} \in N_{J, j}$ be the generators of $C_{J, j}$ which correspond to the divisors $D_{1}^{Y}, \ldots, D_{\ell}^{Y}$. For $1 \leq s \leq k, 1 \leq t \leq \ell$, define $a_{s t}$ to be the coefficient of $D_{s}^{X}$ of the divisor $\mu^{*}\left(D_{t}^{Y}\right)$. Then we define $\nu\left(e_{s}\right)=\sum a_{s t} v_{t}$. Note that if $\left(X, \Sigma_{X}\right) \rightarrow\left(Y, \Sigma_{Y}\right)$ is a smooth toric morphism of toric varieties, then $\nu: \Sigma_{X} \rightarrow \Sigma_{Y}$ is the natural morphism of polyhedral complexes.

We have the following proposition relating $\nu$ to $\mu$ :

Proposition 5. If $C=C_{J, j} \subset \Sigma_{Y}$, then $\nu^{-1} C$ is the union of fans $\Sigma_{\alpha} \subset \Sigma_{X}$ with the following properties:

(1): $\Sigma_{\alpha}$ is a refinement of $C$ obtained by adding finitely-many 1-dim rays.

(2): The lattice $N_{\alpha}$ of $\Sigma_{\alpha}$ is a finite index sub-lattice of $N_{C}$.

(3): The fans $\Sigma_{\alpha}$ are in $1-1$ correspondence with connected components $U_{\alpha}$ of $\mu^{-1}\left(N_{Y_{J, j}^{o}}\right)$. The map $U_{\alpha} \rightarrow N_{Y_{J, j}^{o}}$ is a fibration given by the smooth toric morphism $\mathbf{P}_{\Sigma_{\alpha}, N_{\alpha}} \rightarrow \mathbf{P}_{C, N_{C}}$ along the fiber, and a $d_{\alpha}=d\left(\Sigma_{\alpha}\right)$-cover of $Y_{J, j}^{o}$ along the base.

For a proof, see [7].

5.3. Pushforward formula for Polyhedral Complexes. Motivated by the description of the push-forward $\nu_{*}$ for toric morphisms, define $\nu_{*}: \mathbb{C}\left[\Sigma_{X}\right] \rightarrow \mathbb{C}\left[\Sigma_{Y}\right]$ as follows. Let $C \subset \Sigma_{Y}$ be an $n$-dimensional cone with dual linear forms $x_{1}^{C}, \ldots, x_{n}^{C}$. Then for $f \in \mathbb{C}\left[\Sigma_{X}\right]$, we define:

$$
\left(\nu_{*} f\right)_{C}=\sum_{\alpha} d_{\alpha} \sum_{C_{i} \in \Sigma_{\alpha}} f_{C_{i}} \cdot \frac{\prod_{j=1}^{n} x_{j}^{C}}{\prod_{j=1}^{n} x_{j}^{C_{i}}} .
$$

The second sum is taken over the cones $C_{i} \subset \Sigma_{\alpha}$ with the same dimension as $C$.

Let $V$ be the toric variety $\bigsqcup_{\alpha} d_{\alpha} \cdot \mathbf{P}_{\Sigma_{\alpha}, N_{\alpha}}$ with polyhedral fan $\Sigma_{V}$. We have a natural toric morphism $V \rightarrow \mathbb{C}^{n}$. We can compactify $V$ and $\mathbb{C}^{n}$ to obtain a smooth toric morphism $\bar{V} \rightarrow \mathbb{P}^{n}$. If we view $f$ as a piece-wise polynomial function on the fan of $\bar{V}$, then the above formula simply corresponds to $\left(\nu_{*} f\right)_{C}$ where $\nu: \Sigma_{\bar{V}} \rightarrow \Sigma_{\mathbb{P}^{n}}$. This identification allows us to apply the tools of the previous section toward the study of $\nu_{*}$.

We first observe that $\left(\nu_{*} f\right)_{C}$ is indeed a polynomial function. This follows from the above identification of $\nu_{*}$ with the equivariant pushforward of a toric morphism. 
Furthermore, if we define $\nu^{*}: \mathbb{C}\left[\Sigma_{Y}\right] \rightarrow \mathbb{C}\left[\Sigma_{X}\right]$ by the formula $\nu^{*}(f)=f \circ \nu$ then the projection formula:

$$
\nu_{*}\left(f \nu^{*} g\right)_{C}=\nu_{*}(f)_{C} \cdot g_{C}
$$

follows from the projection formula in equivariant cohomology.

Proposition 6. $\nu_{*}(f)$ is a piece-wise polynomial function.

Proof. We first show that $\nu_{*}\left(f^{C}\right)$ is piece-wise polynomial.

Fix $f=f^{C}$. Suppose $\nu(C) \subset C_{0}$ for some $C_{0} \subset \Sigma_{Y}$ of dimension $k=\operatorname{dim} C$. Then $\nu_{*}(f)_{C_{0}}=d\left(\Sigma_{C_{0}}\right) \prod_{j=1}^{k} x_{j}^{C_{0}}$. Suppose $C_{1}$ is a cone containing $C_{0}$. We wish to show $\left(\nu_{*} f\right)_{C_{1}}$ is an extension of $\left(\nu_{*} f\right)_{C_{0}}$.

Consider the toric morphism $\sigma: \mathbf{P}_{\Sigma_{C_{1}}, N\left(\Sigma_{C_{1}}\right)} \rightarrow \mathbb{C}^{\operatorname{dim} C_{1}}$ induced by the map

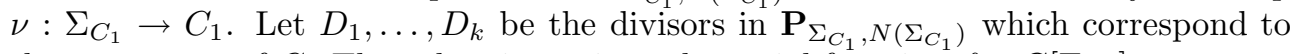
the generators of $C$. Then the piece-wise polynomial function $f \in \mathbb{C}\left[\Sigma_{C_{1}}\right]$ represents the equivariant Thom class of $D_{1} \cap \cdots \cap D_{k}$. Since $\sigma\left(D_{1} \cap \cdots \cap D_{k}\right)$ is the affine subspace of $\mathbb{C}^{\operatorname{dim} C_{1}}$ corresponding to $C_{0}$, we have that $\sigma_{*}(f)$ is the degree of $\sigma$ along $D_{1} \cap \cdots \cap D_{k}$ times the polynomial function which represents the equivariant Thom class of this subspace. But this implies that:

$$
\nu_{*}(f)_{C_{1}}=d\left(\Sigma_{C_{1}}\right) \frac{\left[N\left(\Sigma_{C_{1}}\right): N\left(C_{1}\right)\right]}{\left[N\left(\Sigma_{C_{0}}\right): N\left(C_{0}\right)\right]} \prod_{j=1}^{k} x_{j}^{C_{0}}=d\left(\Sigma_{C_{0}}\right) \prod_{j=1}^{k} x_{j}^{C_{0}} .
$$

We need to explain the last equality. If $C_{0}$ corresponds to the strata $Y_{I, j}^{o}$ and $U \rightarrow$ $N_{Y_{I, j}^{o}}$ is the fibration in Proposition 5 corresponding to the subdivision $\Sigma_{C_{0}}$, then $d\left(\Sigma_{C_{0}}\right)\left[N\left(\Sigma_{C_{0}}\right): N\left(C_{0}\right)\right]$ and $d\left(\Sigma_{C_{1}}\right)\left[N\left(\Sigma_{C_{1}}\right): N\left(C_{1}\right)\right]$ both give the number of points in the pre-image of a generic point in $N_{Y_{I, j}^{o}}$.

Next suppose that $C$ is mapped to a cone $C_{0}$ of strictly larger dimension. Consider the toric morphism $\mathbf{P}_{\Sigma_{C_{0}}, N\left(\Sigma_{C_{0}}\right)} \rightarrow \mathbf{P}_{C_{0}, N\left(C_{0}\right)}$ induced by the map $\nu: \Sigma_{C_{0}} \rightarrow C_{0}$. The polynomial function $f \in \mathbb{C}\left[\Sigma_{C_{0}}\right]$ represents the Thom class of an exceptional toric subvariety. Thus $\nu_{*}(f)=0$, and it is easy to verify that $\nu_{*}(f)=0$ on every cone containing $C_{0}$. Thus, $\nu_{*}$ maps the elements $f^{C}$ to piecewise polynomial functions. Since these functions generate $\mathbb{C}\left[\Sigma_{X}\right]$ as a $\mathbb{C}\left[\Sigma_{Y}\right]$-module, the proposition follows from the projection formula. $\square$

In what follows we assume that $\mu: X \rightarrow Y$ is an equivariant map of projective $T$ spaces. Furthermore, we assume that the irreducible components of $D_{X}$ and $D_{Y}$ are invariant under the $T$-action. Define a map $\rho_{X}: \mathbb{C}\left[\Sigma_{X}\right] \rightarrow H_{T}^{*}(X)$ as follows: Fix a cone $C=C_{I, i}$ which corresponds to a connected component of the intersection locus of the divisors $D_{1}, \ldots, D_{k}$. Define $\rho_{X}\left[f^{C} \cdot\left(f^{D_{1}}\right)^{a_{1}} \ldots\left(f^{D_{k}}\right)^{a_{k}}\right]=\Phi_{X_{I, i}} \wedge D_{1}^{a_{1}} \wedge \ldots \wedge D_{k}^{a_{k}}$. Here $\Phi_{X_{I, i}}$ denotes the equivariant Thom class of $X_{I, i} \subset X$ and, by abuse of notation, $D_{j}$ denote the equivariant Thom classes of the divisors $D_{j}$. Note that the map $\rho_{X}$ is intended to be an equivariant cohomology analogue to the map $\rho: \mathbb{C}\left[\Sigma_{X}\right] \rightarrow A^{*}(X)$ in [7], where $A^{*}(X)$ is the Chow ring of $X$. In that situation, Borisov and Libgober defined $\rho$ by sending $f^{C} \cdot\left(f^{D_{1}}\right)^{a_{1}} \ldots\left(f^{D_{k}}\right)^{a_{k}}$ to $X_{I, i} \cap\left(D_{1}\right)^{a_{1}} \ldots \cap\left(D_{k}\right)^{a_{k}}$. It is natural to view the equivariant Thom classes of $X_{I, i}$, etc, as the equivariant cohomology analogues of these Chow classes.

LEMMA 2. $\rho_{X}$ is a ring homomorphism.

Proof. Fix cones $C_{1}=C_{I_{1}, i_{1}}$ and $C_{2}=C_{I_{2}, i_{2}}$. It suffices to prove the theorem for the polynomials $f^{C_{1}}$ and $f^{C_{2}}$. Let $I=I_{1} \cup I_{2}$. Let $C_{I, i}$ denote the cones which 
correspond to components of the intersection $X_{I_{1}, i_{1}} \cap X_{I_{2}, i_{2}}$. Clearly

$$
f^{C_{1}} f^{C_{2}}=\sum_{I, i} f^{C_{I, i}} \prod_{I_{1} \cap I_{2}} f^{D_{j}}
$$

Thus $\rho_{X}\left(f^{C_{1}} f^{C_{2}}\right)=\sum_{I, i} \Phi_{X_{I, i}} \prod_{I_{1} \cap I_{2}} D_{j}$. However, by the equivariant version of the excess intersection formula, this is precisely the formula for $\rho_{X}\left(f^{C_{1}}\right) \rho_{X}\left(f^{C_{2}}\right)$.

\section{LEMMA 3. $\rho_{X} \nu^{*}=\mu^{*} \rho_{Y}$.}

Proof. It suffices to check this for polynomials $f^{C_{I, k}}$. If $D$ is a divisor on $Y$ whose irreducible components are components of $D_{Y}$, then $\nu^{*} f^{D}$ is the piecewise linear function corresponding to $\mu^{*} D$. It follows that $\rho_{X} \nu^{*} f^{D}=\mu^{*} \rho_{Y} f^{D}$. Since all the maps are ring homomorphisms, this implies that $\rho_{X} \nu^{*} \prod_{j \in I} f^{D_{j}}=\mu^{*} \rho_{Y} \prod_{j \in I} f^{D_{j}}$. Let $\mu^{*} D_{i}=\sum_{j} a_{i j} E_{j}$ as Cartier divisors. As in the lemma in the Appendix, choose equivariant Thom forms $\Phi_{E_{j}}$ and $\Phi_{D_{i}}$ with support in small tubular neighborhoods of their respective divisors so that:

$$
\mu^{*} \Phi_{D_{i}}=\sum_{j} a_{i j} \Phi_{E_{j}}+d \psi_{i}
$$

as forms. Here $\psi_{i}$ are equivariant forms with compact support in $\mu^{-1} N_{D_{i}}$. Let $\{I, k\}$ index the connected components of $\cap_{I} D_{i}$. If we choose $N_{D_{i}}$ sufficiently small, then

$$
\prod_{I} \Phi_{D_{i}}=\sum_{I, k}\left(\prod_{I} \Phi_{D_{i}}\right)_{I, k}
$$

where $\left(\prod_{I} \Phi_{D_{i}}\right)_{I, k}$ is the extension by zero of the form $\left.\prod_{I} \Phi_{D_{i}}\right|_{N_{I, k}}$.

Now $\prod_{I} f^{D_{i}}=\sum f^{C_{I, k}}$ and clearly $\left(\prod_{I} \Phi_{D_{i}}\right)_{I, k}$ is a representative of $\rho_{Y}\left(f^{C_{I, k}}\right)$. We have that

$$
\mu^{*}\left(\prod_{I} \Phi_{D_{i}}\right)_{I, k}=\left\{\prod_{I}\left(\sum_{j} a_{i j} \Phi_{E_{j}}+d \psi_{i}\right)\right\}_{\mu^{-1} N_{I, k}}
$$

where the subscript $\mu^{-1} N_{I, k}$ means the extension by zero of the form restricted to this open set. Since the $\psi_{i}$ forms have compact support in $\mu^{-1} N_{D_{i}}$, this form is cohomologous to

$$
\left\{\prod_{I} \sum_{j} a_{i j} \Phi_{E_{j}}\right\}_{\mu^{-1} N_{I, k}}
$$

But this is in turn a representative of $\rho_{X} \nu^{*} f^{C_{I, k}}$.

LEMma 4. $\mu_{*} \rho_{X}=\rho_{Y} \nu_{*}$.

Proof. Since $\rho_{X} \nu^{*}=\mu^{*} \rho_{Y}$ and the polynomials $f^{C}$ generate $\mathbb{C}\left[\Sigma_{X}\right]$ as a $\mathbb{C}\left[\Sigma_{Y}\right]$ module, by the projection formula it suffices to check $\mu_{*} \rho_{X} f^{C}=\rho_{Y} \nu_{*} f^{C}$.

Case 1: $C_{I, i}$ is mapped by $\nu$ to a cone $C_{J, j}$ of the same dimension.

From the proof of Proposition 4, $\nu_{*} f^{C_{I, i}}=d f^{C_{J, j}}$ where $d$ is the degree of $\mu$ : $X_{I, i} \rightarrow Y_{J, j}$. Thus, $\rho_{Y} \nu_{*} f^{C_{I, i}}=d \Phi_{Y_{J, j}}=\mu_{*} \nu_{*} f^{C_{I, i}}$.

Case 2: $C_{I, i}$ is mapped by $\nu$ into a cone of strictly larger dimension.

As shown in Proposition $4, \nu_{*} f^{C_{I, i}}=0$, so $\rho_{Y} \nu_{*} f^{C_{I, i}}=0=\mu_{*} \Phi_{X_{I, i}}=\mu_{*} \rho_{X} f^{C_{I, i}}$. 
REMARK 2. It is clear using the formalism of section 2 that the above lemmas relating $\mu$ to $\nu$ extend without difficulty to the ring $\mathbb{C}\left[\left[\Sigma_{X}\right]\right]$ of piecewise convergent power series. In this situation, $\rho_{X}$ is a map from $\mathbb{C}\left[\left[\Sigma_{X}\right]\right]$ into $\widehat{H}(X)$.

6. Elliptic Genera and Toroidal Morphisms. Let $\left(\hat{X}, \sum_{I_{\hat{X}}} \hat{D}_{j}\right)$ and $\left(X, \sum_{I_{X}} D_{i}\right)$ be smooth projective toroidal embeddings with $T$-actions which leave $\hat{D}_{j}$ and $D_{i}$ invariant. Let $G$ be a finite group which acts toroidally on $\hat{X}$ and commutes with the action of $G$. Suppose that $\mu: \hat{X} \rightarrow X$ is a $T$-equivariant toroidal morphism which is birational to a global quotient by $G$. If $\alpha_{i}$ are coefficients of the irreducible components $D_{i}$, define $\beta_{j}$ so that $\mu^{*}\left(K_{X}+\sum \alpha_{i} D_{i}\right)=K_{\hat{X}}+\sum \beta_{j} \hat{D}_{j}$. Then:

Theorem 7.

$$
\mu_{*} \mathcal{E} l l_{o r b}\left(\hat{X}, \sum \beta_{j} \hat{D}_{j}, G\right)=\mathcal{E} l l\left(X, \sum \alpha_{i} D_{i}\right)
$$

Proof. The proof of this theorem follows almost word for word the proof of Theorem 5.1 in [7]. The only difference is that here we examine the equivariant pushforward of equivariant cohomology classes, whereas [7] examine the push-forward of (non-equivariant) classes in the Chow ring. We reproduce the proof here for completeness.

We refer frequently to the notation in the previous section: For commuting elements $g, h \in G$, let $\hat{X}_{\gamma}^{g, h}$ denote the $\gamma$-th fixed component of $(g, h)$. Since the action of $G$ on $\hat{X}$ is toroidal, $\hat{X}_{\gamma}^{g, h}$ may be identified with $X_{I^{g, h}, i}$ for some indexing set $I^{g, h} \subset I_{\hat{X}}$.

Consider the following class in $\widehat{H}(\hat{X})$ :

$$
\begin{gathered}
E=\frac{1}{|G|} \sum_{g h=h g ; \hat{X}_{\gamma}^{g, h}} \Phi_{\hat{X}_{\gamma}^{g, h}}^{T} \cdot \prod_{I_{\hat{X}^{-}}-I_{\gamma}^{g, h}} \frac{\frac{\hat{D}_{j}}{2 \pi i} \theta\left(\frac{\hat{D}_{j}}{2 \pi i}-\left(-\beta_{j}+1\right) z\right) \theta^{\prime}(0)}{\theta\left(\frac{\hat{D}_{j}}{2 \pi i}\right) \theta\left(-\left(-\beta_{j}+1\right) z\right)} . \\
\prod_{I_{\gamma}^{g, h}} \frac{\theta\left(\frac{\hat{D}_{j}}{2 \pi i}+\epsilon_{j}(g)-\epsilon_{j}(h) \tau-\left(-\beta_{j}+1\right) z\right) \frac{\theta^{\prime}(0)}{2 \pi i}}{\theta\left(\frac{\hat{D}_{j}}{2 \pi i}+\epsilon_{j}(g)-\epsilon_{j}(h) \tau\right) \theta\left(-\left(-\beta_{j}+1\right) z\right)} e^{2 \pi i\left(-\beta_{j}+1\right) \epsilon_{j}(h) z} .
\end{gathered}
$$

Here $\Phi_{\hat{X}_{\gamma}^{g, h}}^{T}$ is the equivariant Thom class of the subvariety $\hat{X}_{\gamma}^{g, h}$, and $\epsilon_{j}(g)$, etc., are defined as in the definition of the orbifold elliptic genus.

Our first goal is to prove

$$
\mu_{*} E=\prod_{I_{X}} \frac{\frac{D_{i}}{2 \pi i} \theta\left(\frac{D_{i}}{2 \pi i}-\left(-\alpha_{i}+1\right) z\right) \theta^{\prime}(0)}{\theta\left(\frac{D_{i}}{2 \pi i}\right) \theta\left(-\left(-\alpha_{i}+1\right) z\right)}
$$

To prove the above equality, we express both sides as the image under $\rho_{\hat{X}}$ and $\rho_{X}$ of piece-wise convergent power series, and apply the push-forward formula from the previous section. To that end, let $F$ be the piece-wise convergent power series defined as follows: Let $C_{\gamma}^{g, h}$ be the cone which corresponds to $X_{\gamma}^{g, h}$. For each cone $C=C_{J, j}$ containing $C_{\gamma}^{g, h}$, let $\left.F_{\gamma}^{g, h}\right|_{C}=$ 


$$
\frac{1}{|G|} \prod_{J} \frac{\frac{x_{j}^{C}}{2 \pi i} \theta\left(\frac{x_{j}^{C}}{2 \pi i}+\epsilon_{j}(g)-\epsilon_{j}(h) \tau-\left(-\beta_{j}+1\right) z\right) \theta^{\prime}(0)}{\theta\left(\frac{x_{j}^{C}}{2 \pi i}+\epsilon_{j}(g)-\epsilon_{j}(h) \tau\right) \theta\left(-\left(-\beta_{j}+1\right) z\right)} e^{2 \pi i\left(-\beta_{j}+1\right) \epsilon_{j}(h) z} .
$$

For cones $C$ not containing $C_{\gamma}^{g, h}$, we define $\left.F_{\gamma}^{g, h}\right|_{C}=0$. In the above expression, $x_{j}^{C}$ are the piece-wise linear functions dual to the generators of $C$. If $\hat{D}_{j}$ are the divisors which correspond to the generators of $C$, then $\epsilon_{j}(g)$, etc., are the infinitesimal weights attached to the divisors, and $\beta_{j}$ are the coefficients of $\hat{D}_{j}$. It is easy to see that $F_{\gamma}^{g, h}$ is a well-defined piece-wise convergent power series, and that $\rho_{\hat{X}}\left(F_{\gamma}^{g, h}\right)$ is the $X_{\gamma}^{g, h}$-th summand in the expression for $E$. We therefore define $F=\sum_{g h=h g, \gamma} F_{\gamma}^{g, h}$, so that $\rho_{\hat{X}}(F)=E$.

Similarly, define $H \in \mathbb{C}\left[\left[\Sigma_{X}\right]\right]$ to be the piece-wise convergent power series:

$$
\left.H\right|_{C}=\prod_{i=1}^{\operatorname{dim} C} \frac{\frac{x_{i}^{C}}{2 \pi i} \theta\left(\frac{x_{i}^{C}}{2 \pi i}-\left(-\alpha_{i}+1\right) z\right) \theta^{\prime}(0)}{\theta\left(\frac{x_{i}^{C}}{2 \pi i}\right) \theta\left(-\left(-\alpha_{i}+1\right) z\right)} .
$$

Clearly $\rho_{X}(H)$ is equal to the right-hand side of 3 . We are therefore reduced to proving that $\nu_{*} F=H$.

Let $C \subset \Sigma_{X}$ be a cone, and let $\Sigma_{\alpha}$ be the subdivisions of $C$ lying in $\Sigma_{\hat{X}}$ which get mapped to $C$ under $\nu$. Let $N_{\alpha}$ denote the lattices of $\Sigma_{\alpha}$, and $N_{C}$ the lattice of $\Sigma_{C}$. Referring to the notation of section 5.3, the formula for $\nu_{*}$ tells us that:

$$
\left(\nu_{*} F\right)_{C}=\left.\sum_{\alpha} d_{\alpha} \sum_{C_{j} \subset \Sigma_{\alpha}} F\right|_{C_{j}} \frac{\prod_{i=1}^{\operatorname{dim} C} x_{i}^{C}}{\prod_{i=1}^{\operatorname{dim} C} x_{i}^{C_{j}}} .
$$

For each $C_{j} \subset \Sigma_{\alpha}$ with the same dimension as $C$, note that $\left.F_{\gamma}^{g, h}\right|_{C_{j}} \neq 0$ if and only if $C_{\gamma}^{g, h} \subset C_{j}$, i.e., if and only if $g$ and $h$ are elements of the group $G_{\alpha}=N_{C} / N_{\alpha}$. We may therefore write the push-forward of $F$ as:

$$
\begin{aligned}
& \prod_{i=1}^{\operatorname{dim} C} x_{i}^{C} \sum_{\alpha} \frac{d_{\alpha}}{|G|} \sum_{g, h \in G_{\alpha}} \\
& \sum_{C_{j} \subset \Sigma_{\alpha}} \frac{\theta\left(\frac{x_{i} C_{j}}{2 \pi i}+\epsilon_{i}(g)-\epsilon_{i}(h) \tau-\left(-\beta_{i}+1\right) z\right) \frac{\theta^{\prime}(0)}{2 \pi i}}{\theta\left(\frac{x_{i}^{C_{j}}}{2 \pi i}+\epsilon_{i}(g)-\epsilon_{i}(h) \tau\right) \theta\left(-\left(-\beta_{i}+1\right) z\right)} e^{2 \pi i\left(-\beta_{i}+1\right) z .}
\end{aligned}
$$

By lemma 8.1 in [7], this is equal to $\left.\sum_{\alpha} \frac{d_{\alpha}\left|G_{\alpha}\right|}{|G|} H\right|_{C}$. But since $\sum_{\alpha} d_{\alpha}\left|G_{\alpha}\right|$ describes the number of points in the pre-image of a generic point in a tubular neighborhood of the closed stratum corresponding to $C$, the coefficient in front of $\left.H\right|_{C}$ in the above expression is 1 . This completes the proof of 3 . To complete the proof, we apply the projection formula together with the following lemma relating the Chern classes of $\hat{X}$ to those of $X$ :

LEMMA 5.

$$
\frac{c(T \hat{X})_{T}}{\mu^{*} c(T X)_{T}}=\frac{\prod_{I_{\hat{X}}}\left(1+c_{1}\left(\hat{D}_{j}\right)_{T}\right)}{\prod_{I_{X}}\left(1+c_{1}\left(D_{i}\right)_{T}\right)}
$$


in $H_{T}^{*}(\hat{X})_{l o c}$

For details, see [7]. The above lemma may be proved using an argument analogous to the proof of lemma 5 in [18]. $\mathrm{u}$

7. Deformation to the Normal Cone. If $Q$ is a holomorphic function of one variable in a neighborhood of the origin, then $Q$ determines a map $\varphi_{Q}: K_{T}(\cdot) \rightarrow \widehat{H}(\cdot)$ by the rule $E \mapsto \prod_{i} Q\left(e_{i}\right)$, where $e_{i}$ represent the equivariant Chern roots of $E$. If, in addition, $Q(0)=1$, then $\varphi_{Q}$ is multiplicative in the sense that $\varphi_{Q}\left(E_{1} \oplus E_{2}\right)=$ $\varphi_{Q}\left(E_{1}\right) \varphi_{Q}\left(E_{2}\right)$.

More generally, let $H$ be a finite abelian group with characters $\{\lambda\}$. Let $\left\{f_{\lambda}\right\}$ be a collection of a holomorphic functions of one variable in a neighborhood of the origin for each character $\lambda$. Let $E$ be a $T \times H$ vectorbundle over a manifold $X$, and suppose that the action of $H$ on $X$ is trivial. Then $E$ decomposes into a direct sum of character subbundles $E=\bigoplus_{\lambda} E_{\lambda}$. The collection $\left\{f_{\lambda}\right\}$ therefore defines a map $\psi: E=\bigoplus_{\lambda} E_{\lambda} \mapsto \prod_{\lambda} \prod_{i} f_{\lambda}\left(e_{\lambda, i}\right)$, where $e_{\lambda, i}$ denote the $T$-equivariant Chern roots of the $T$-vectorbundle $E_{\lambda}$. Let us fix a multiplicative map $\varphi=\varphi_{Q}$ and a (possibly not multiplicative) map $\psi=\psi_{\left\{f_{\lambda}\right\}}$.

Let $X$ be a compact $T \times H$-variety and let $Z$ be a smooth $T \times H$-invariant subvariety. Let $V$ be a connected component of $X^{H}$. In the proof of the lemma below, the only difficult case to examine is when $Z \cap V \equiv W$ is a proper subset of $V$. We therefore assume this throughout. Let $\pi: \widetilde{X} \rightarrow X$ be the blow-up of $X$ along $Z$ and let $\widetilde{V}$ be the proper transform of $V$. Clearly $N_{\tilde{V} / \tilde{X}}$ has the same $H$-character decomposition as $N_{V / X}$. We may therefore make sense of $\psi\left(N_{\tilde{V} / \tilde{X}}\right)$. The goal of this section is to prove the following crucial technical lemma relating $\varphi(T \widetilde{V}) \psi\left(N_{\tilde{V} / \tilde{X}}\right)$ to $\varphi(T V) \psi\left(N_{V / X}\right)$.

Lemma 6.

$$
\pi_{*} \varphi(T \widetilde{V}) \psi\left(N_{\tilde{V} / \tilde{X}}\right)-\varphi(T V) \psi\left(N_{V / X}\right)=\left(i_{W}\right)_{*} \Theta
$$

Here $i_{W}$ is the inclusion map and $\Theta \in \widehat{H}(W)$ is a universal class which depends only on the data of $W, N_{W / V}, i_{W}^{*} N_{Z / X}, \varphi$, and $\psi$.

The argument given below is an adaptation of the argument in [17] which was given for the non-equivariant case with $\psi=1$.

Proof. Let $\Pi: M_{X} \rightarrow X \times \mathbb{P}^{1}$ be the blow-up along $Z \times\{\infty\}$. We give $M_{X}$ the obvious $T \times H$ action. Let $M_{V}$ be the proper transform of $V \times \mathbb{P}^{1}$. Clearly $M_{V} \rightarrow V \times \mathbb{P}^{1}$ is the blow-up along $W \times\{\infty\}$. It is easy to see that $N_{M_{V} / M_{X}} \in$ $K_{T}\left(M_{V}\right) \otimes R(H)$ has the same $H$-character decomposition as $N_{V / X}$.

Define $N_{0}$ to be the sub-bundle of $i_{W}^{*} N_{Z / X}$ on which $H$-acts trivially. Let $N_{1}=$ $i_{W}^{*} N_{Z / X} / N_{0}$. Then $i_{W}^{*} T X$ decomposes as $T W \oplus N_{W / Z} \oplus N_{0} \oplus N_{1}$. Clearly $T W \oplus N_{0}=$ $i_{W}^{*} T V$, and therefore $N_{V / W}=N_{0}$ is a sub-bundle of $i_{W}^{*} N_{Z / X}$ with quotient $N_{1}$.

Let $g: M_{X} \rightarrow \mathbb{P}^{1}$ be the composition $M_{X} \rightarrow X \times \mathbb{P}^{1} \rightarrow \mathbb{P}^{1}$. Then $\operatorname{div}(g)=$ $X-\widetilde{X}-\mathbb{P}\left(N_{Z / X} \oplus 1\right)$. Furthermore, $\operatorname{div}\left(\left.g\right|_{M_{V}}\right)=V-\widetilde{V}-\mathbb{P}\left(N_{W / V} \oplus 1\right)$. Let $i_{V}, i_{\tilde{V}}$, and $i_{\mathbb{P}\left(N_{W / V} \oplus 1\right)}$ denote the respective inclusion maps of these divisors in $M_{V}$.

Let $p: \mathbb{P}\left(N_{W / V} \oplus 1\right) \rightarrow W$ be the obvious fibration, and let $S$ denote the tautological bundle over $\mathbb{P}\left(N_{W / V} \oplus 1\right)$. 
CLAIM.

$$
\begin{aligned}
i_{V}^{*} N_{M_{V} / M_{X}} & =N_{V / X} \\
i_{\widetilde{V}}^{*} N_{M_{V} / M_{X}} & =N_{\tilde{V} / \tilde{X}} \\
i_{\mathbb{P}\left(N_{W / V} \oplus 1\right)}^{*} N_{M_{V} / M_{X}} & =p^{*} N_{W / Z} \oplus p^{*} N_{1} \otimes S^{*}
\end{aligned}
$$

(1) is obvious. To prove (2), note first that $\left.T M_{X}\right|_{\widetilde{V}}$ decomposes as $T \widetilde{V} \oplus N_{\widetilde{V} / \widetilde{X}} \oplus$ $\left.N_{\tilde{X} / M_{X}}\right|_{\tilde{V}}$ and also as $\left.T \widetilde{V} \oplus N_{\widetilde{V} / M_{V}} \oplus N_{M_{V} / M_{X}}\right|_{\widetilde{V}}$. Then simply notice that in both decompositions, $N_{\tilde{V} / \tilde{X}}$ and $i_{\widetilde{V}}^{*} N_{M_{V} / M_{X}}$ are the nontrivial $H$-eigenspaces.

To prove (3), note that $\left.T M_{X}\right|_{\mathbb{P}\left(N_{W / V} \oplus 1\right)}$ decomposes in the following two ways:

$$
\begin{aligned}
& \left.T M_{X}\right|_{\mathbb{P}\left(N_{W / V} \oplus 1\right)}= \\
& T \mathbb{P}\left(N_{W / V} \oplus 1\right) \oplus \mathcal{O}(-1)_{\mathbb{P}\left(N_{W / V} \oplus 1\right)} \oplus i_{\mathbb{P}\left(N_{W / V} \oplus 1\right)}^{*} N_{M_{V} / M_{X}}= \\
& T \mathbb{P}\left(N_{W / V} \oplus 1\right) \oplus N_{\mathbb{P}\left(N_{W / V} \oplus 1\right) / \mathbb{P}\left(N_{Z / X} \oplus 1\right)} \oplus i_{\mathbb{P}\left(N_{W / V}^{*} \oplus 1\right)} \mathcal{O}(-1)_{\mathbb{P}\left(N_{Z / X} \oplus 1\right)}
\end{aligned}
$$

Observing $i_{\mathbb{P}\left(N_{W / V} \oplus 1\right)}^{*} \mathcal{O}(-1)_{\mathbb{P}\left(N_{Z / X} \oplus 1\right)}=\mathcal{O}(-1)_{\mathbb{P}\left(N_{W / V} \oplus 1\right)}$ it follows that $i_{\mathbb{P}\left(N_{W / V} \oplus 1\right)}^{*} N_{M_{V} / M_{X}}=N_{\mathbb{P}\left(N_{W / V} \oplus 1\right) / \mathbb{P}\left(N_{Z / X} \oplus 1\right)}$. It is easy to verify that this bundle in turn is equal to $p^{*} N_{W / Z} \oplus p^{*} N_{1} \otimes S^{*}$.

Since $\operatorname{div}\left(\left.g\right|_{M_{V}}\right)=V-\widetilde{V}-\mathbb{P}\left(N_{W / V} \oplus 1\right)$, as equivariant classes $V=\widetilde{V}+\mathbb{P}\left(N_{W / V} \oplus\right.$ 1). Let $u$ be the equivariant Thom class of $\widetilde{V}$ (that is, the Thom class of its normal bundle), and let $v$ be the equivariant Thom class of $\mathbb{P}\left(N_{W / V} \oplus 1\right)$. Then $u+v$ is the equivariant Thom class of $V$. Since $V$ is disjoint from $\widetilde{V}$ and $\mathbb{P}\left(N_{W / V} \oplus 1\right)$, we have the relations $u(u+v)=v(u+v)=0$. Note also that $u v$ is the equivariant Thom class of $\mathbb{P}\left(N_{W / V}\right)$, which is the exceptional divisor of $\widetilde{V} \rightarrow V$.

Let $f$ be the holomorphic function in a neighborhood of the origin which satisfies the relation $Q(z)=\frac{z}{f(z)}$. Then by the above claim:

$$
\begin{aligned}
& \varphi\left(T M_{V}\right) \psi\left(N_{M_{V} / M_{X}}\right) f(u+v)=\left(i_{V}\right)_{*} \varphi(T V) \psi\left(N_{V / X}\right) \\
& \varphi\left(T M_{V}\right) \psi\left(N_{M_{V} / M_{X}}\right) f(u)=\left(i_{\widetilde{V}}\right)_{*} \varphi(T \widetilde{V}) \psi\left(N_{\widetilde{V} / \tilde{X}}\right) \\
& \varphi\left(T M_{V}\right) f(v)=\left(i_{\mathbb{P}\left(N_{W / V} \oplus 1\right)}\right)_{*} \varphi\left(T \mathbb{P}\left(N_{W / V} \oplus 1\right)\right) \psi\left(N_{\mathbb{P}\left(N_{W / V} \oplus 1\right) / \mathbb{P}\left(N_{Z / X} \oplus 1\right)}\right)
\end{aligned}
$$

Note that since $u$ and $v$ are equivariant Thom classes, their degree zero part is an element of $C^{\infty}\left(M_{V}\right) \otimes \mathfrak{t}^{*}$ which vanishes at the origin of $\mathfrak{t}$. Hence $f(u)$, etc., are well-defined elements of $\widehat{H}\left(M_{V}\right)$.

Since $f(z)=z+\ldots$, we can define $g=f^{-1}$ in a possibly smaller neighborhood of the origin. Consider the two-variable holomorphic function $h\left(z_{1}, z_{2}\right)=f\left(z_{1}+z_{2}\right)$. Clearly $h\left(z_{1}, z_{2}\right)=h\left(g\left(f\left(z_{1}\right)\right), g\left(f\left(z_{2}\right)\right)\right)$. Define $F\left(y_{1}, y_{2}\right)=h\left(g\left(y_{1}\right), g\left(y_{2}\right)\right)$. Then $F$ is holomorphic in a neighborhood of the origin and $F\left(f\left(z_{1}\right), f\left(z_{2}\right)\right)=f\left(z_{1}+z_{2}\right)$. From [12] we have the formula:

$$
F\left(y_{1}, y_{2}\right) g^{\prime}\left(y_{1}\right) g^{\prime}\left(y_{2}\right)=\sum_{(i, j) \neq(0,0)} \varphi\left(H_{i j}\right) y_{1}^{i} y_{2}^{j} .
$$

Here $\varphi\left(H_{i j}\right)$ is the non-equivariant genus induced by $f$ of the degree $(1,1)$ hypersurface $H_{i j} \subset \mathbb{P}^{i} \times \mathbb{P}^{j}$. If we plug in $f(u)$ and $f(v)$ for $y_{1}$ and $y_{2}$, we get 
$F(f(u), f(v)) g^{\prime}(f(u)) g^{\prime}(f(v))=f(u+v) g^{\prime}(f(u)) g^{\prime}(f(v))$. By the relations $u(u+v)=$ $v(u+v)=0$, this last term is cohomologous to $f(u+v)$. It is instructive to go over this last point in detail. Write $f(x+y)=\sum a_{n}(x+y)^{n}$ and $g^{\prime}(f(x)) g^{\prime}(f(y))=\sum b_{i j} x^{i} y^{j}$. Note that $b_{00}=1$. Let $h_{N I J}=\sum_{n<N} a_{n}(x+y)^{n} \cdot \sum_{i \leq I, j \leq J} b_{i j} x^{i} y^{j}$. Then $h_{N I J} \rightarrow f(x+y) g^{\prime}(f(x)) g^{\prime}(f(y))$ in the $\bar{C}^{\infty}$ topology. Abusing notation, let $u$ and $v$ denote fixed representatives for their respective cohomology classes. We have that $h_{N I J}(u, v)=\sum_{n<N} a_{n}(u+v)^{n}+d \eta_{N I J}$. Evaluating at a sufficiently small $s$ gives: $h_{N I J}(u, v)(s)=\sum_{n<N} a_{n}(u(s)+v(s))^{n}+d \eta_{N I J}(s)$. Taking the limit in the indices $N, I, J$, we get $h(u, v)(s)=f(u(s)+v(s))+d \eta(s)$. We therefore have that $e v(h)=e v(f(u+v))$, and therefore $h=f(u+v)$ in $\widehat{H}\left(M_{V}\right)$.

At the same time, $f(u+v)=\sum_{(i, j) \neq(0,0)} \varphi\left(H_{i j}\right) f(u)^{i} f(v)^{j}$. Thus

$$
\begin{aligned}
& \varphi\left(T M_{V}\right) \psi\left(N_{M_{V} / M_{X}}\right) f(u+v)=\varphi\left(T M_{V}\right) \psi\left(N_{M_{V} / M_{X}}\right) f(u)+ \\
& \varphi\left(T M_{V}\right) \psi\left(N_{M_{V} / M_{X}}\right) f(v)+\varphi\left(T M_{V}\right) \psi\left(N_{M_{V} / M_{X}}\right) \sum_{i+j \geq 2} \varphi\left(H_{i j}\right) f(u)^{i} f(v)^{j}
\end{aligned}
$$

From the relations $u^{2}=-u v$ and $v^{2}=-u v$, we have that $f(u)^{i+1}=f(u) f(-v)^{i}$. Therefore,

$$
\sum_{i+j \geq 2} \varphi\left(H_{i j}\right) f(u)^{i} f(v)^{j}=\sum_{i+j \geq 2, i \geq 1} \varphi\left(H_{i j}\right) f(u) f(-v)^{i-1} f(v)^{j}+\sum_{j \geq 2} \varphi\left(H_{0 j}\right) f(v)^{j}
$$

We therefore have $\sum_{i+j \geq 2} \varphi\left(H_{i j}\right) f(u)^{i} f(v)^{j}=u v \frac{f(u)}{u} G(v)+v J(v)$ for some universal convergent power series $G$ and $J$. Let $\nu=i_{\mathbb{P}\left(N_{W / V} \oplus 1\right)}^{*} v$. Clearly $\nu=c_{1}(S)$. Let $w=\left.\nu\right|_{\mathbb{P}\left(N_{W / V}\right)}$. Finally, for ease of notation, write $N=p^{*} N_{W / Z} \oplus p^{*} N_{1} \otimes S^{*}$.

$$
\begin{aligned}
& \varphi\left(T M_{V}\right) \psi\left(N_{M_{V} / M_{X}}\right) f(u+v)= \\
& \varphi\left(T M_{V}\right) \psi\left(N_{M_{V} / M_{X}}\right) f(u)+\varphi\left(T M_{V}\right) \psi\left(N_{M_{V} / M_{X}}\right) f(v)+ \\
& \varphi\left(T M_{V}\right) \psi\left(N_{M_{V} / M_{X}}\right) u v \frac{f(u)}{u} G(v)+\varphi\left(T M_{V}\right) \psi\left(N_{M_{V} / M_{X}}\right) v J(v)
\end{aligned}
$$

It follows that

$$
\begin{aligned}
& \left(i_{V}\right)_{*} \varphi(T V) \psi\left(N_{V / X}\right)= \\
& \left(i_{\widetilde{V}}\right)_{*} \varphi(T \widetilde{V}) \psi\left(N_{\tilde{V} / \tilde{X}}\right)+\left(i_{\mathbb{P}\left(N_{W / V} \oplus 1\right)}\right)_{*} \varphi\left(T \mathbb{P}\left(N_{W / V} \oplus 1\right)\right) \psi(N)+ \\
& \left(i_{\mathbb{P}\left(N_{W / V}\right)}\right)_{*} \varphi\left(\left.T \mathbb{P}\left(N_{W / V}\right) \oplus S\right|_{\mathbb{P}\left(N_{W / V}\right)}\right) \psi\left(\left.N\right|_{\mathbb{P}\left(N_{W / V}\right)}\right) G(w)+ \\
& \left(i_{\mathbb{P}\left(N_{W / V} \oplus 1\right)}\right)_{*} \varphi\left(T \mathbb{P}\left(N_{W / V} \oplus 1\right) \oplus S\right) \psi(N) J(\nu)
\end{aligned}
$$

Now apply the push-forward $\Pi_{*}$ to the above equation. Note that $\Pi \circ i_{V}$ is the inclusion $v \mapsto(v, 0)$ in $V \times \mathbb{P}^{1}$. $\Pi \circ i_{\widetilde{V}}$ is the composition of the blow-down $\widetilde{V} \rightarrow V$ with the inclusion of $V$ at infinity in $V \times \mathbb{P}^{1}$. From the blow-up diagram:

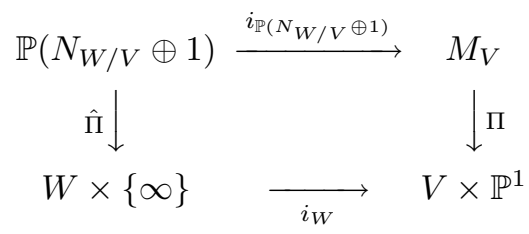


we have that $\Pi \circ i_{\mathbb{P}\left(N_{W / V} \oplus 1\right)}=i_{W} \circ \hat{\Pi}$. Finally, $\Pi \circ i_{\mathbb{P}\left(N_{W / V}\right)}$ is clearly the composition of the blow-down map $\hat{\pi}: \mathbb{P}\left(N_{W / V}\right) \rightarrow W$ with the inclusion $i_{W}$. Therefore, applying the pushforward $\Pi_{*}$ gives the equation:

$$
\begin{aligned}
& \varphi(T V) \psi\left(N_{V / X}\right)= \\
& \pi_{*} \varphi(T \widetilde{V}) \psi\left(N_{\tilde{V} / \tilde{X}}\right)+\left(i_{W}\right)_{*}\left\{\hat{\Pi}_{*} \varphi\left(T \mathbb{P}\left(N_{W / V} \oplus 1\right)\right) \psi(N)+\right. \\
& \hat{\pi}_{*} \varphi\left(\left.T \mathbb{P}\left(N_{W / V}\right) \oplus S\right|_{\mathbb{P}\left(N_{W / V}\right)}\right) \psi\left(\left.N\right|_{\mathbb{P}\left(N_{W / V}\right)}\right) G(w)+ \\
& \left.\hat{\Pi}_{*} \varphi\left(T \mathbb{P}\left(N_{W / V} \oplus 1\right) \oplus S\right) \psi(N) J(\nu)\right\} .
\end{aligned}
$$

Since the term in the curly braces depends only on the data of $i_{W}^{*} N_{Z / X}, N_{W / V}$, and $W$, this proves the lemma.

REMARK 3. Of course in the above proof, if $Q(x)=\frac{\frac{x}{2 \pi i} \theta\left(\frac{x}{2 \pi i}-z\right) \frac{\theta^{\prime}(0)}{2 \pi i}}{\theta\left(\frac{2 \pi}{2 \pi i}\right) \theta(-z)}$ and $f_{\lambda}=$ $\frac{e^{2 \pi i \lambda(h) \tau z} \theta\left(\frac{x}{2 \pi i}+\lambda(g)-\lambda(h) \tau-z\right) \frac{\theta^{\prime}(0)}{2 \pi i}}{\theta\left(\frac{x}{2 \pi i}+\lambda(g)-\lambda(h) \tau\right) \theta(-z)}$, then $\varphi(T V) \psi\left(N_{V / X}\right)$ is the equivariant elliptic class associated to the pair $(g, h)$ and the $(g, h)$-fixed component $V$.

8. The Normal Cone Space. Let $W \subset X$ be a connected $T$-invariant subvariety of a projective $T$-space $X$. Suppose the normal bundle $N_{W / X}$ splits into a composition $L_{1} \oplus \ldots \oplus L_{k}$ of $T$-vectorbundles. Define $p: X^{*} \rightarrow W$ to be the fiber bundle with fiber $p^{-1}(w)=\mathbb{P}\left(L_{1} \oplus 1\right)_{w} \times \ldots \times \mathbb{P}\left(L_{k} \oplus 1\right)_{w}$. It is easy to see that $X^{*}$ contains a copy of $W$ with the same normal bundle $N_{W / X}$. In our proof of the blow-up formula, we will ultimately reduce all computations on $X$ to computations on the more manageable space $X^{*}$. We therefore devote this section to gathering some important facts about the topology of $X^{*}$.

If we give the trivial vectorbundle 1 the trivial action, then the action of $T$ on $W$ lifts naturally to $X^{*}$. Give $L_{i}$ a metric so that $T$ acts on $L_{i}$ by isometries, and give $\mathbb{P}\left(L_{i} \oplus 1\right)$ the induced metric.

Define vectorbundles $Q_{i} \rightarrow X^{*}$ as follows: For $w \in W$ and $\left(\ell_{1}, \ldots, \ell_{k}\right)$ lines in $\left(L_{1} \oplus 1\right)_{w}, \ldots,\left(L_{k} \oplus 1\right)_{w}$, define $\left(Q_{i}\right)_{\left(w, \ell_{1}, \ldots, \ell_{k}\right)}=\ell_{i}^{\perp} \subset\left(L_{i} \oplus 1\right)_{w}$. These bundles inherit natural $T$-actions. Observe furthermore that $i_{W}^{*}\left(Q_{i}\right)$ is naturally isomorphic to $L_{i}$.

Define $V_{i} \subset X^{*}$ to be the subvariety $\left\{\left(w, \ell_{1}, \ldots, \ell_{k}\right): \ell_{i}=[0: 1]\right\}$. For $i=$ $1, \ldots, k, V_{i}$ are connected $T$-subvarieties, with connected intersection locus, and $W=$ $V_{1} \cap \ldots \cap V_{k}$.

Finally, let $f: \widetilde{X^{*}} \rightarrow X^{*}$ denote the blow-up of $X^{*}$ along $Z=V_{1} \cap \ldots \cap V_{j}$ with exceptional divisor $E$. We have the following intersection-theoretic result:

Theorem 8. $c_{\text {top }}\left(f^{*} Q_{1} \oplus \ldots \oplus f^{*} Q_{j} \otimes \mathcal{O}(-E)\right)_{T}=0$.

Proof. We will show that $\operatorname{Hom}\left(L, f^{*} Q_{1} \oplus \ldots \oplus f^{*} Q_{j}\right)$ has an equivariant global nowhere zero section, where $L$ is a line bundle with the same equivariant first Chern class as $\mathcal{O}(E)$.

We first give an explicit construction of the line bundle $L$. Let $0 \leq t_{1}, \ldots, t_{j}$. Define $S_{t_{1}, \ldots, t_{j}} \subset \widetilde{X^{*}}$ to be the subset $\left\{\left(w,\left[v_{1}: 1\right], \ldots,\left[v_{j}: 1\right],\left[v_{1}: \ldots: v_{j}\right], \bar{\ell}\right):\right.$ $\left.\left\|v_{i}\right\|=t_{i}\right\}$. Here $\bar{\ell}$ represents a point in $\mathbb{P}\left(L_{j+1} \oplus 1\right)_{w} \times \ldots \times \mathbb{P}\left(L_{k} \oplus 1\right)_{w}$. We will refer to points in $\widetilde{X^{*}}$ which are not contained in any $S_{t_{1}, \ldots, t_{j}}$ as points at infinity. Let $\rho:[0, \infty) \rightarrow \mathbb{R}$ be a bump function equal to zero in the region $[0,1 / 3)$ and equal to one in the region $[2 / 3, \infty)$. For $0 \leq t_{i} \leq 1$ and $v=\left(w,\left[v_{1}: 1\right], \ldots,\left[v_{j}: 1\right],\left[v_{1}: \ldots: v_{j}\right], \bar{\ell}\right)$ 
a point in $S_{t_{1}, \ldots, t_{j}}$, let $L_{v} \subset\left(L_{1} \oplus 1\right)_{w} \oplus \ldots \oplus\left(L_{j} \oplus 1\right)_{w}$ be the span of the vector $\tilde{v}=\left(\left(1-\rho\left(t_{1}\right)\right) v_{1}, \rho\left(t_{1}\right), \ldots,\left(1-\rho\left(t_{j}\right)\right) v_{j}, \rho\left(t_{j}\right)\right)$. Outside this set, we define $L_{v}$ to be the span of the vector $0 \oplus 1 \oplus \ldots \oplus 0 \oplus 1$. This clearly defines a smooth line bundle on $\widehat{X}^{*}$ with the same equivariant first Chern class as $\mathcal{O}(E)$.

We now prove that $\operatorname{Hom}\left(L, f^{*} Q_{1} \oplus \ldots \oplus f^{*} Q_{j}\right)$ has a global equivariant nowhere zero section. For $v_{i} \in L_{i}$, define:

$$
h_{i}\left(v_{i}\right)=\left\{\begin{array}{cc}
\left(-v_{i},\left\|v_{i}\right\|^{2}\right) & \left\|v_{i}\right\| \leq 1 \\
\left(-\frac{v_{i}}{\left\|v_{i}\right\|^{2}}, 1\right) & \left\|v_{i}\right\| \geq 1
\end{array}\right.
$$

For $v=\left(w,\left[v_{1}: 1\right], \ldots,\left[v_{j}: 1\right],\left[v_{1}: \ldots: v_{j}\right], \bar{\ell}\right) \in S_{t_{1}, \ldots, t_{j}}$, we define $L_{v} \rightarrow$ $\left(Q_{1} \oplus \ldots \oplus Q_{j}\right)_{f(v)}$ by $\tilde{v} \mapsto\left(h_{1}\left(v_{1}\right), \ldots, h_{j}\left(v_{j}\right)\right)$. This section extends in a natural way to the points at infinity, giving us a continuous nowhere zero equivariant section $s_{0}$ of $\operatorname{Hom}\left(L, f^{*} Q_{1} \oplus \ldots \oplus f^{*} Q_{j}\right)$. The section is only continuous because the function:

$$
h(x)= \begin{cases}x^{2} & |x| \leq 1 \\ \frac{1}{x^{2}} & |x| \geq 1\end{cases}
$$

is not smooth. However, we may remedy this by approximating our continuous section $s_{0}$ by a smooth section and then averaging over the group $T$. Since $s_{0}$ was nowhere zero and fixed by the $T$-averaging process, the new smooth section will remain nowhere zero after averaging over $T$.

REMARK 4 . The intuition behind the preceding theorem is that if $Z=D_{1} \cap \ldots \cap$ $D_{j}$ is the complete intersection of a collection of normal crossing divisors, then the proper transforms $\widetilde{D}_{i}$ of $D_{i}$ are disjoint when we blow up along $Z$. We therefore have that $c_{t o p}\left(\mathcal{O}\left(\widetilde{D}_{1}\right) \oplus \ldots \oplus \mathcal{O}\left(\widetilde{D}_{j}\right)\right)_{T}=0$. While the above theorem is known, the proof given here will be useful later.

One easily observes that $i_{V_{i}}^{*} Q_{i}=N_{V_{i} / X^{*}}$. We might expect, therefore, that $c_{\text {top }}\left(Q_{i}\right)_{T}$ was the equivariant Thom class of $V_{i}$. This is in fact the case, as the following lemma proves:

Lemma 7. $c_{t o p}\left(Q_{i}\right)_{T}=i_{V_{i *}} 1$

Proof. By the naturality properties of the equivariant Chern classes, it is enough to prove the above lemma for $X^{*}=\mathbb{P}(L \oplus 1)$, where $L$ is a $T$-vectorbundle over $W$, and $Q$ is the universal quotient bundle over $\mathbb{P}(L \oplus 1)$. Here, $W$ itself plays the role of $V_{i}$ in the statement of the lemma. As above, we endow the trivial bundle 1 with the trivial $T$-action.

Let us first prove that $c_{t o p}\left(Q_{i}\right)=i_{W *} 1$ in the non-equivariant category. Let $p: \mathbb{P}(L \oplus 1) \rightarrow W$ denote the obvious projection map. Let $r=\operatorname{rk}(Q)$. From the exact sequence:

$$
0 \rightarrow S \rightarrow p^{*}(L \oplus 1) \rightarrow Q \rightarrow 0
$$

we have that $c_{r}(Q)=\left(\frac{p^{*} c(L)}{1-c_{1}\left(S^{*}\right)}\right)_{r}$, where $(\cdot)_{r}$ denotes the degree $r$ part. Let $p_{w}$ : $\mathbb{P}(L \oplus 1)_{w} \rightarrow w$ denote the restriction of $p$ to the fiber over $w$. Then $\left.c_{r}(Q)\right|_{\mathbb{P}(L \oplus 1)_{w}}=$ $\left.c_{1}\left(S^{*}\right)^{r}\right|_{\mathbb{P}(L \oplus 1)_{w}}$, which clearly integrates to 1 over the fiber.

We next observe that $Q$ has a global no-where zero section away from $W$. We define this section as follows: $s:(w,[v: z]) \mapsto\left(w,[v: z], \frac{\bar{z} v}{\|v\|^{2}},-1\right)$ We therefore have 
that $c_{r}(Q)$ is exact away from $W$. Hence, by subtracting off an exact form, we may represent $c_{r}(Q)$ by a form which has compact support in a tubular neighborhood of $W$, and which integrates to one along every fiber of the tubular neighborhood. It follows that $c_{r}(Q)=i_{W *} 1$ at least in the non-equivariant sense.

In general, $c_{r}(Q)_{T}$ is at least an equivariant extension of the Thom class of $W$. Moreover, $c_{r}(Q)_{T}$ is equivariantly exact outside $W$. We prove this as follows: Observe first that the non-zero section we defined on the complement of $W$ was in fact equivariant. This section therefore induces a splitting $Q=Q^{\prime} \oplus \mathbb{C}$ outside of $W$. Since we endowed 1 with the trivial $T$-action, one may easily verify that the $\mathbb{C}$ in the above splitting inherits a trivial action as well. It follows that $c_{r}(Q)_{T}$ is equivariantly exact outside of $W$.

By subtracting off an exact form, we get that $c_{r}(Q)_{T}$ may be represented by an equivariant form which has compact support in a tubular neighborhood of $W$, and which integrates to one along every fiber of the tubular neighborhood. It follows that $c_{r}(Q)_{T}$ is the equivariant Thom class of $W$.

We next prove a formula relating the equivariant Chern class of the blow-up of $X^{*}$ along $Z$ to that of $X^{*}$. Note first that $T X^{*}$ splits holomorphically and equivariantly into a direct sum of sub-bundles $F \oplus M$, with $i_{Z}^{*} F=T Z$ and $i_{Z}^{*} M=N_{Z / X^{*}}$. We may therefore apply the following lemma to compare the equivariant Chern classes of $X^{*}$ and $\mathrm{Bl}_{Z} X^{*}$.

Lemma 8. Let $Y$ be a complex $T$-space, and $Z \subset Y$ a T-invariant complex submanifold. Suppose that $T Y$ splits holomorphically and equivariantly into a direct sum of sub-bundles $F \oplus M$ such that $i_{Z}^{*} F=T Z$ and $i_{Z}^{*} M=N_{Z / Y}$. Let $f: \widetilde{Y} \rightarrow Y$ be the blow-up of $Y$ along $Z$ with exceptional divisor $E$. Then:

$$
c(T \widetilde{Y})_{T}=c\left(f^{*} F\right)_{T} c\left(f^{*} M \otimes \mathcal{O}(-E)\right)_{T} c(\mathcal{O}(E))_{T}
$$

in the $\operatorname{ring} H_{T}^{*}(\widetilde{Y})_{l o c}$.

Proof. By localization, it suffices to prove the equality at every fixed component in $\widetilde{Y}$. Let $\widetilde{P} \subset \widetilde{Y}$ be a fixed component which is the proper transform of a fixed component $P \subset Y$. If $P$ is disjoint from $Z$, then the equality of (4) at $\widetilde{P} \cong P$ is trivial. Otherwise, $\widetilde{P}$ is equal to the blow-up of $P$ at $P \cap Z$. Note that $i_{P}^{*} F$ decomposes as $F_{0} \oplus F_{1}$, where $T$ acts trivially on $F_{0}$ and nontrivially on $F_{1}$. Similarly, $i_{P}^{*} M=M_{0} \oplus M_{1}$. Clearly $T P=F_{0} \oplus M_{0}$ and $N_{P / Y}=F_{1} \oplus M_{1}$.

Applying $i_{\widetilde{P}}^{*}$ to $(4)$, we have:

$$
\begin{aligned}
i_{\widetilde{P}}^{*}(\mathrm{LHS})= & c(T \widetilde{P}) c\left(N_{\widetilde{P} / \widetilde{Y}}\right)_{T} \\
i_{\widetilde{P}}^{*}(\mathrm{RHS})= & c\left(f^{*} F_{0}\right) c\left(f^{*} M_{0} \otimes \mathcal{O}(-E)\right) c\left(i_{\widetilde{P}}^{*} \mathcal{O}(E)\right) \\
& c\left(f^{*} F_{1}\right)_{T} c\left(f^{*} M_{1} \otimes \mathcal{O}(-E)\right)_{T}
\end{aligned}
$$

Since $\widetilde{P}$ is the blow-up of $P$ along $P \cap Z$ and $i_{P \cap Z}^{*} M_{0}=N_{P \cap Z / P}$, the relation $c(T \widetilde{P})=c\left(f^{*} F_{0}\right) c\left(f^{*} M_{0} \otimes \mathcal{O}(-E)\right) c\left(i_{\widetilde{P}}^{*} \mathcal{O}(E)\right)$ is well-known (see [10]). It suffices therefore to prove that $c\left(N_{\widetilde{P} / \tilde{Y}}\right)_{T}=i_{\widetilde{P}}^{*}\left(c\left(f^{*} F_{1}\right)_{T} c\left(f^{*} M_{1} \otimes \mathcal{O}(-E)\right)_{T}\right)$. To this end we prove the following claim:

CLAIM. $N_{\widetilde{P} / \widetilde{Y}} \cong i_{\widetilde{P}}^{*}\left(f^{*} F_{1} \oplus f^{*} M_{1} \otimes \mathcal{O}(-E)\right)$ as T-vectorbundles.

To prove this, consider $f$ as a map $f: \widetilde{P} \rightarrow P$. For simplicity of notation, write $E$ for $E \cap \widetilde{P}$. View $N_{\widetilde{P} / \widetilde{Y}}$ as a sheaf, i.e., $N_{\widetilde{P} / \widetilde{Y}}(U)=\Gamma\left(U, N_{\widetilde{P} / \widetilde{Y}}\right)$. The 
derivative $D f: N_{\tilde{P} / \tilde{Y}} \rightarrow f^{*} N_{P / Y}=f^{*} F_{1} \oplus f^{*} M_{1}$ is a sheaf map, and maps onto the subsheaf $f^{*} F_{1} \oplus f^{*} M_{1}(-E)$. Here $f^{*} M_{1}(-E)$ represents the subsheaf of $f^{*} M_{1}$ corresponding to sections of $f^{*} M_{1}$ which vanish along $E$. Let $s_{0}$ denote the global section of $\mathcal{O}(E)$ induced by the defining equations of $E$. Then $\otimes s_{0}^{-1}: f^{*} M_{1}(-E) \rightarrow$ $f^{*} M_{1} \otimes \mathcal{O}(-E)$ is a sheaf isomorphism. Define $\beta=\left(i d \oplus \otimes s_{0}^{-1}\right) \circ D f$. By computing in local coordinates, one verifies easily that $\beta: N_{\widetilde{P} / \widetilde{Y}} \rightarrow f^{*} F_{1} \oplus f^{*} M_{1} \otimes \mathcal{O}(-E)$ is a sheaf isomorphism. Furthermore, for $s$ a local section of $N_{\widetilde{P} / \widetilde{Y}}, s(p)=0$ if and only if $(\beta s)(p)=0$. Therefore, beta induces an isomorphism of the corresponding vectorbundles. Moreover, since $\beta$ is clearly equivariant, the vectorbundle isomorphism is equivariant.

This completes the proof if $P \cap E<P$. Next, suppose that $\widetilde{P} \subset E$. For this case, it suffices to prove the following fact:

CLAIM. $i_{E}^{*}(T \tilde{Y} \oplus \mathbb{C})=i_{E}^{*}\left(f^{*} F \oplus f^{*} M \otimes \mathcal{O}(-E) \oplus \mathcal{O}(E)\right)$.

We prove this as follows: Let $\pi: \mathbb{P}\left(N_{Z / Y}\right) \rightarrow Z$ be the natural projection, and let $S$ denote the tautological bundle over $\mathbb{P}\left(N_{Z / Y}\right)$. Then $i_{E}^{*} T \tilde{Y}=T \mathbb{P}\left(N_{Z / Y}\right) \oplus S$.

Since $i_{E}^{*} f^{*}=\pi^{*} i_{Z}^{*}$, we have that $i_{E}^{*} f^{*} F=\pi^{*} i_{Z}^{*} F=\pi^{*} T Z$ and $i_{E}^{*} f^{*} M=$ $\pi^{*} N_{Z / Y}$. Thus,

$$
i_{E}^{*}\left(f^{*} F \oplus f^{*} M \otimes \mathcal{O}(-E) \oplus \mathcal{O}(E)\right)=\pi^{*} T Z \oplus \pi^{*} N_{Z / Y} \otimes S^{*} \oplus S .
$$

From the exact sequence $0 \rightarrow S \rightarrow \pi^{*} N_{Z / Y} \rightarrow Q \rightarrow 0$, we have that $\pi^{*} N_{Z / Y} \otimes$ $S^{*}=\mathbb{C} \oplus Q \otimes S^{*}$, where $Q$ is the tautological quotient bundle. The claim then follows from the observation that $T \mathbb{P}\left(N_{Z / Y}\right)=\pi^{*} T Z \oplus Q \otimes S^{*}$. This completes the proof of the lemma.

REMARK 5 . Note that if $\widetilde{Y}$ is equivariantly formal, the above proof implies that (4) holds in the unlocalized ring $H_{T}^{*}(\widetilde{Y})$.

We may rewrite the left-hand side of (4) as $c\left(f^{*} T Y\right)_{T} c\left(f^{*} M\right)_{T}^{-1} c\left(f^{*} M \otimes\right.$ $\mathcal{O}(-E))_{T} c(\mathcal{O}(E))_{T}$. Viewed as an element of $\widehat{H}(\widetilde{Y})$, it is easy to verify that this expression remains the same if we replace $M$ by any bundle $M^{\prime}$ with $i_{Z}^{*} M^{\prime}=N_{Z / Y}$. We therefore obtain the following corollary pertaining to $X^{*}$ :

Corollary 1. Let $f: \widetilde{X^{*}} \rightarrow X^{*}$ be the blow-up of $X^{*}$ along $Z=V_{1} \cap \ldots \cap V_{j}$ for $j \leq k$ with exceptional divisor $E$. Then the following formula holds in $\widehat{H}\left(\widetilde{X^{*}}\right)$ :

$$
c\left(T \widetilde{X^{*}}\right)_{T}=\frac{c\left(f^{*} T X^{*}\right)_{T}}{\prod_{i=1}^{j} c\left(f^{*} Q_{i}\right)_{T}} \prod_{i=1}^{j} c\left(f^{*} Q_{i} \otimes \mathcal{O}(-E)\right)_{T} c(\mathcal{O}(E))_{T}
$$

We end this section with a technical lemma which is the blow-up analogue of lemma 7.

Lemma 9. For $1 \leq i \leq k$, let $\widetilde{V}_{i}$ be the proper transform of $V_{i}$ under the above blow-up: $f: \widetilde{X}^{*} \rightarrow X^{*}$. Then $c_{\text {top }}\left(f^{*} Q_{i} \otimes \mathcal{O}(-E)\right)_{T}$ is the equivariant Thom class of $\widetilde{V}_{i}$ for $1 \leq i \leq j$, and $c_{\text {top }}\left(f^{*} Q_{i}\right)_{T}$ is the equivariant Thom class of $\widetilde{V}_{i}$ for $j+1 \leq i \leq k$. Moreover $i_{\widetilde{V}_{i}}^{*} c\left(f^{*} Q_{i} \otimes \mathcal{O}(-E)\right)_{T}=c\left(N_{\widetilde{V}_{i} / \widetilde{X}^{*}}\right)_{T}$ for $1 \leq i \leq j$ and $i_{\widetilde{V}_{i}}^{*} c\left(f^{*} Q_{i}\right)_{T}=$ $c\left(N_{\widetilde{V}_{i} / \widetilde{X^{*}}}\right)_{T}$ for $j+1 \leq i \leq k$.

Proof. Let $1 \leq i \leq j$. Recall the equivariant continuous nowhere vanishing section $s_{0}$ of $\operatorname{Hom}\left(L, f^{*} Q_{1} \oplus \ldots \oplus f^{*} Q_{k}\right)$ constructed in the proof of theorem 8. Let 
$\pi_{i}$ denote the projection from $f^{*} Q_{1} \oplus \ldots \oplus f^{*} Q_{k}$ onto the $i$-th factor. Then $\pi_{i} \circ s_{0}$ is an equivariant section of $f^{*} Q_{i} \otimes L^{*}$. From the construction of $s_{0}$, it is clear that $s_{0}$ vanishes precisely along $\widetilde{V}_{i}$. Hence, the equivariant top Chern class of $f^{*} Q_{i} \otimes L^{*}$ is the equivariant Thom class of $\widetilde{V}_{i}$. Since $L$ has the same equivariant first Chern class as $\mathcal{O}(E)$, this proves $c_{\text {top }}\left(f^{*} Q_{i} \otimes \mathcal{O}(-E)\right)_{T}=i_{\widetilde{V}_{i}} 1$.

To prove the second part of the lemma for $1 \leq i \leq j$, apply $i_{\widetilde{V}_{i}}^{*}$ to both sides of the equation in corollary 1 . The LHS becomes $c\left(T \widetilde{V}_{i}\right)_{T} c\left(N_{\widetilde{V}_{i} / \widetilde{X}^{*}}\right)_{T}$ while the RHS becomes:

$$
\begin{aligned}
& \frac{c\left(f^{*} N_{V_{i} / X^{*}} \otimes \mathcal{O}\left(-E \cap \widetilde{V}_{i}\right)\right)_{T} c\left(f^{*} T V_{i}\right)_{T}}{\prod_{\ell \neq i}^{j} c\left(f^{*} N_{V_{\ell} / X^{*}}\right)_{T}} \times \\
& \prod_{\ell \neq i}^{j} c\left(f^{*} N_{V_{\ell} / X^{*}} \otimes \mathcal{O}\left(-E \cap \widetilde{V}_{i}\right)\right)_{T} c\left(\mathcal{O}\left(E \cap \widetilde{V}_{i}\right)\right)_{T} .
\end{aligned}
$$

Here we have used the fact that $i_{V_{\ell}}^{*} Q_{\ell}=N_{V_{\ell} / X^{*}}$. By corollary 1, the factor multiplying $i_{\widetilde{V}_{i}}^{*} c\left(f^{*} Q_{i} \otimes \mathcal{O}(-E)\right)_{T}=c\left(f^{*} N_{V_{i} / X^{*}} \otimes \mathcal{O}\left(-E \cap \widetilde{V}_{i}\right)\right)_{T}$ in the above expression is equal to $c\left(T \widetilde{V}_{i}\right)_{T}$. We therefore have that $i_{\widetilde{V}_{i}}^{*} c\left(f^{*} Q_{i} \otimes \mathcal{O}(-E)\right)_{T}=c\left(N_{\widetilde{V}_{i} / \widetilde{X}^{*}}\right)_{T}$.

Next, let $j+1 \leq i \leq k$. From the proof of lemma 7 we know that $Q_{i}$ has an equivariant nowhere zero section in the complement of $V_{i}$. Pulling back this section by $f$ gives an equivariant nowhere zero section in the complement of $f^{-1} V_{i}=\widetilde{V}_{i}$. It follows that $c_{t o p}\left(f^{*} Q_{i}\right)_{T}$ must be localized in a neighborhood of $\widetilde{V}_{i}$. By the equivariant Thom isomorphism, $c_{t o p}\left(f^{*} Q_{i}\right)_{T}$ must be a complex number multiple of the equivariant Thom class of $\widetilde{V}_{i}$. But since $f_{*} c_{t o p}\left(f^{*} Q_{i}\right)_{T}$ is the Thom class of $V_{i}$, this complex number multiple must be equal to one.

The proof of the second part of the lemma for $j+1 \leq i \leq k$ is analogous to the proof given above for $1 \leq i \leq j$. $\mathrm{\square}$

9. Twisted Polyhedral Complex. Throughout, we assume the following:

(a) $X$ is a smooth projective variety with a holomorphic $T \times H$ action, where $T$ is a torus and $H$ is a finite abelian group.

(b) $Z \subset X$ is a $T$-invariant smooth subvariety.

(c) $V \subset X^{H}$ is a connected component.

(d) $W=V \cap Z$ is connected. (see remark at the end of this section)

As noted above, $i_{W}^{*} N_{Z / X}$ splits as $N_{0} \oplus N_{1}$, where $N_{0}$ denotes the sub-bundle of $i_{W}^{*} N_{Z / X}$ on which $H$ acts trivially. Furthermore, $N_{1}$ decomposes as a sum of subbundles $\oplus N_{\lambda}$ corresponding to the characters of the $H$-action on $N_{1}$. Finally, $N_{W / Z}$ also splits into character sub-bundles $\oplus N_{\varepsilon}$.

Let $E_{1}, \ldots, E_{\ell}, D_{1}, \ldots, D_{k}$ be smooth normal crossing divisors on $X$ intersecting $Z$ normally and non-trivially. We label these divisors so that $i_{W}^{*} \mathcal{L}_{D_{i}} \hookrightarrow N_{0}$ and $i_{W}^{*} \mathcal{L}_{E_{j}} \hookrightarrow N_{1}$. Write $i_{W}^{*} \mathcal{L}_{D_{i}}=\Delta_{i}$ and $i_{W}^{*} \mathcal{L}_{E_{j}}=\xi_{j}$. Write $N_{0}=F_{0} \oplus \bigoplus \Delta_{i}$ and $N_{1}=\bigoplus F_{\lambda} \oplus \bigoplus \xi_{j}$.

Define a new space $X^{*}$ as follows: $p: X^{*} \rightarrow W$ is the fiber bundle with fiber $p^{-1}(w)=$

$$
\begin{aligned}
& \prod \mathbb{P}\left(N_{\varepsilon} \oplus 1\right)_{w} \times \mathbb{P}\left(F_{0} \oplus 1\right)_{w} \times \\
& \prod \mathbb{P}\left(\Delta_{i} \oplus 1\right)_{w} \times \prod \mathbb{P}\left(F_{\lambda} \oplus 1\right)_{w} \times \prod \mathbb{P}\left(\xi_{j} \oplus 1\right)_{w}
\end{aligned}
$$


Clearly $X^{*}$ contains a copy of $W$ with normal bundle $N_{W / X}$. Moreover, as in the previous section, each of the bundles $N_{\varepsilon}, F_{0}, F_{\lambda}, \Delta_{i}$, and $\xi_{j}$ are the restrictions to $W$ of global equivariant bundles $Q_{N_{\varepsilon}}, Q_{F_{0}}, Q_{F_{\lambda}}, Q_{\Delta_{i}}$ and $Q_{\xi_{j}}$. Recall also that the top Chern classes of these bundles are Poincaré dual to varieties $V_{N_{\varepsilon}}, V_{F_{0}}, V_{F_{\lambda}}, V_{\Delta_{i}}$, and $V_{\xi_{j}}$. The analogue of $Z$ in $X^{*}$ is the intersection locus of the varieties $V_{F_{0}}, V_{F_{\lambda}}, V_{\Delta_{i}}$, and $V_{\xi_{j}}$. We will continue to call this intersection locus $Z$.

We associate a polyhedral complex with integral structure $\left(\Sigma, N_{\Sigma}\right)$ to $X^{*}$ as follows: Let $s_{\varepsilon}=\operatorname{rk}\left(N_{\varepsilon}\right), r=\operatorname{rk}\left(F_{0}\right)$, and let $r_{\lambda}=\operatorname{rk}\left(F_{\lambda}\right)$. Let $w^{\varepsilon, a}, x^{b}, y^{\lambda, c}, d^{i}$, and $e^{j}$ be an integral basis for $N_{\Sigma}=\mathbb{Z}^{\sum_{\varepsilon} s_{\varepsilon}+r+\sum_{\lambda} r_{\lambda}+k+\ell}$, for $a=1, \ldots, s_{\varepsilon}, b=1, \ldots, r$, $c=1, \ldots, r_{\lambda}, i=1, \ldots, k$, and $j=1, \ldots, \ell$. Define $\Sigma$ to be the cone in the first orthant of the vectorspace $N_{\Sigma} \otimes \mathbb{R}$. Let $w_{\varepsilon, a}, x_{b}, y_{\lambda, c}, d_{i}$, and $e_{j}$ be the linear forms on this vectorspace which are dual to above basis vectors. Then $\mathbb{C}[\Sigma]=\mathbb{C}\left[w_{\varepsilon, a}, x_{b}, y_{\lambda, c}, d_{i}, e_{j}\right]$. Let $G=\prod_{\varepsilon} S_{s_{\varepsilon}} \times S_{r} \times \prod_{\lambda} S_{r_{\lambda}}$, where $S_{n}$ denotes the symmetric group in $n$ letters. Then $G$ acts on $\mathbb{C}[\Sigma]$ by permuting the linear forms $w_{\varepsilon, a}, x_{b}, y_{\lambda, c}$ in the obvious manner. Consider the following correspondence:

$$
\begin{aligned}
w_{\varepsilon, a} & \longleftrightarrow T \text {-Chern roots of } Q_{N_{\varepsilon}} \\
x_{b} & \longleftrightarrow T \text {-Chern roots of } Q_{F_{0}} \\
y_{\lambda, c} & \longleftrightarrow T \text {-Chern roots of } Q_{F_{\lambda}} \\
d_{i} & \longleftrightarrow c_{1}\left(Q_{\Delta_{i}}\right)_{T} \\
e_{j} & \longleftrightarrow c_{1}\left(Q_{\xi_{j}}\right)_{T}
\end{aligned}
$$

Such a correspondence defines a natural map $\rho: \mathbb{C}[\Sigma]^{G} \rightarrow H_{T}^{*}\left(X^{*}\right)$.

Let $\phi: \widetilde{X^{*}} \rightarrow X^{*}$ denote the blow-up of $X^{*}$ along $Z$ with exceptional divisor $E$. Define $\widetilde{\Sigma}$ to be the polyhedral complex obtained from $\Sigma$ by adding the ray through the vector $\sum x_{b}+\sum y_{\lambda, c}+\sum d_{i}+\sum e_{j}$. As before, let $w_{\varepsilon, a}, x_{b}, y_{\lambda, c}, d_{i}$, and $e_{j}$ denote the linear forms on $\mathbb{C}[\widetilde{\Sigma}]$ which are dual to vectors $w^{\varepsilon, a}, x^{b}, y^{\lambda, c}, d^{i}$, and $e^{j}$. Let $t$ be the linear form dual to $\sum x_{b}+\sum y_{\lambda, c}+\sum d_{i}+\sum e_{j}$. Then:

$$
\mathbb{C}[\widetilde{\Sigma}] \cong \mathbb{C}\left[t, w_{\varepsilon, a}, x_{b}, y_{\lambda, c}, d_{i}, e_{j}\right] / \prod_{b, c, \lambda, i, j} x_{b} y_{\lambda, c} d_{i} e_{j} .
$$

$G$ acts on $\mathbb{C}[\widetilde{\Sigma}]$ in the obvious manner. Consider the correspondence:

$$
\begin{aligned}
w_{\varepsilon, a} & \longleftrightarrow T \text {-Chern roots of } \phi^{*} Q_{N_{\varepsilon}} \\
x_{b} & \longleftrightarrow T \text {-Chern roots of } \phi^{*} Q_{F_{0}} \otimes \mathcal{O}(-E) \\
y_{\lambda, c} & \longleftrightarrow T \text {-Chern roots of } \phi^{*} Q_{F_{\lambda}} \otimes \mathcal{O}(-E) \\
d_{i} & \longleftrightarrow c_{1}\left(\phi^{*} Q_{\Delta_{i}} \otimes \mathcal{O}(-E)\right)_{T} \\
e_{j} & \longleftrightarrow c_{1}\left(\phi^{*} Q_{\xi_{j}} \otimes \mathcal{O}(-E)\right)_{T}
\end{aligned}
$$

By theorem 8 , this correspondence induces a well-defined homomorphism $\rho: \mathbb{C}[\widetilde{\Sigma}]^{G} \rightarrow$ $H_{T}^{*}\left(X^{*}\right)$.

It is easy to see that $\nu^{*}: \mathbb{C}[\Sigma]^{G} \rightarrow \mathbb{C}[\widetilde{\Sigma}]^{G}$ and similarly $\nu_{*}: \mathbb{C}[\widetilde{\Sigma}]^{G} \rightarrow \mathbb{C}[\Sigma]^{G}$. We have the following important lemmas:

LEMMA 10.

$$
\begin{aligned}
& \rho \nu^{*}=\phi^{*} \rho \\
& \phi_{*} \rho=\rho \nu_{*}
\end{aligned}
$$


Proof. For notational convenience, let $\bar{w}_{\varepsilon, a}, \bar{x}_{b}, \ldots$ denote the $T$-Chern roots corresponding to $w_{\varepsilon, a}, x_{b}, \ldots$ With this notation, $\rho(f)\left(w_{\varepsilon, a}, x_{b}, \ldots\right)$ is equal to $f\left(\bar{w}_{\varepsilon, a}, \bar{x}_{b}, \ldots\right)$. We first prove 8: We have:

$$
\begin{aligned}
\phi^{*} \rho(f) & =f\left(\phi^{*} \bar{w}_{\varepsilon, a}, \phi^{*} \bar{x}_{b}, \ldots\right) \\
& =f\left(\bar{w}_{\varepsilon, a}, \bar{x}_{b}+\bar{t}, \ldots\right)=f\left(\rho\left(w_{\varepsilon, a}\right), \rho\left(x_{b}+t\right), \ldots\right) \\
& =f\left(\rho \nu^{*} w_{\varepsilon, a}, \rho \nu^{*} x_{b}, \ldots\right)=\rho \nu^{*} f
\end{aligned}
$$

8 allows us to reduce the proof of 9 to the case where $f=t^{n}$. Let $N=\operatorname{codim} Z$. For $n<N-1, \nu_{*} t^{n}=0=\phi_{*} \rho(t)^{n}$, so the claim is true in this case. Otherwise, for $n=(\ell-1+j), \phi_{*} \rho\left(x_{0}^{n}\right)=(-1)^{j-1}\left(i_{Z}\right)_{*} s_{j}\left(N_{Z / X^{*}}\right)_{T}=(-1)^{j-1}\left(i_{Z}\right)_{*} i_{Z}^{*} s_{j}(M)_{T}=$ $(-1)^{j-1} s_{j}(M)_{T} c_{\text {top }}(M)_{T}$. Here $s_{j}(M)_{T}$ denotes the $j$-th equivariant Segre class of the bundle $M=Q_{F_{0}} \oplus \bigoplus_{\lambda} Q_{F_{\lambda}} \oplus \bigoplus Q_{\Delta_{i}} \oplus \bigoplus_{j} Q_{\xi_{j}}$. The last equality follows from lemma 7. Clearly this last expression is equal to $\rho(P)$ for some universal polynomial $P$ in the Chern roots of $M$. We are therefore reduced to proving that $\nu_{*}(f)=P$. Let $s=\operatorname{rank}\left(N_{W / Z}\right)$ and let $\mu: \widetilde{\mathbb{C}^{s+N}} \rightarrow \mathbb{C}^{s+N}$ denote the blow-up of $\mathbb{C}^{s+N}$ along $\mathbb{C}^{s} \times 0$, where we give both spaces the structure of toric varieties, with big torus $L$. $\mathbb{C}[\widetilde{\Sigma}]$ and $\mathbb{C}[\Sigma]$ both correspond to the rings of piece-wise polynomial functions on the fans of $\widetilde{\mathbb{C}^{s+N}}$ and $\mathbb{C}^{s+N}$, which are in turn isomorphic to the $L$-equivariant cohomology rings of the above spaces. We may view $\nu_{*}$ as the polyhedral version of the equivariant pushforward $\mu_{*}: H_{L}^{*}\left(\widetilde{\mathbb{C}^{s+N}}\right) \rightarrow H_{L}^{*}\left(\mathbb{C}^{s+N}\right)$. Then $\nu_{*} f=P$ follows from the fact that $\mu_{*}\left(c_{1}(E)_{L}\right)^{N-1+j}=P$, where here we evaluate $P$ at the equivariant Chern classes of the coordinate hyperplanes.

REMARK 6. As in section 5.3, the above theorems hold for piece-wise convergent power series.

10. Blow-up Formula for Orbifold Elliptic Genus. Let $X$ be a smooth projective variety with a holomorphic $G \times T$ action, $D=\sum_{I} \alpha_{i} D_{i}$ a $G \times T$-invariant $G$-normal crossing divisor with coefficients $\alpha_{i}<1, g, h \in G$ a pair of commuting elements, and $X_{\gamma}^{g, h}$ a component of the common fixed point locus of $g$ and $h$. Recall the definition given in section 3 for the orbifold elliptic class of the pair $\left(X_{\gamma}^{g, h}, D\right)$. The goal in this section is to prove the following theorem:

ThEOREM 9. Let $f: \widetilde{X} \rightarrow X$ be the blow-up along a smooth $G \times T$-invariant subvariety $Z$ which has normal crossings with respect to the components of D. Fix a commuting pair $g, h \in G$ and a component $X_{\gamma}^{g, h}$ of the fixed point locus $X^{g, h}$. Let $\left\{\tilde{X}_{\mu}^{g, h}\right\}$ denote the components of $\tilde{X}^{g, h}$ which get mapped to $X_{\gamma}^{g, h}$ under $f$. Then:

$$
f_{*} \sum_{\mu} \mathcal{E} l l_{\text {orb }}\left(\widetilde{X}_{\mu}^{g, h}, \widetilde{D}\right)=\mathcal{E} l l_{o r b}\left(X_{\gamma}^{g, h}, D\right)
$$

Here $\widetilde{D}$ is the divisor satisfying $f^{*}\left(K_{X}+D\right)=K_{\widetilde{X}}+\widetilde{D}$.

Proof. By the projection formula, it suffices to assume that every component of $D$ intersects $Z$ with multiplicity 1 . Furthermore, by applying deformation to the normal cone (lemma 6) we may assume that $X$ is the normal cone space $X^{*}$ described in 
section 9. Using the notation from section 9, we have the following correspondences:

$$
\begin{aligned}
H & \longleftrightarrow(g, h) \\
Z \cap X_{\gamma}^{g, h} & \longleftrightarrow W \\
\left\{D_{j}\right\}_{j \in I_{\gamma}^{g, h}} & \longleftrightarrow\left\{V_{\xi_{j}}\right\} \\
\left\{D_{i}\right\}_{i \notin I_{\gamma}^{g, h}} & \longleftrightarrow\left\{V_{\Delta_{i}}\right\} \\
X_{\gamma}^{g, h} & \longleftrightarrow \bigcap_{\varepsilon} V_{N_{\varepsilon}} \cap \bigcap_{\lambda} V_{F_{\lambda}} \cap \bigcap_{j} V_{\xi_{j}} \\
Z & \longleftrightarrow V_{F_{0}} \cap \bigcap_{\lambda} V_{F_{\lambda}} \cap \bigcap_{i} V_{\Delta_{i}} \cap \bigcap_{j} V_{\xi_{j}} .
\end{aligned}
$$

Given these identifications, $\mathcal{E} l l_{o r b}\left(X_{\gamma}^{g, h}, D\right)=$

$$
\begin{aligned}
& \prod_{T X^{*}} \frac{\frac{x_{i}}{2 \pi i} \theta\left(\frac{x_{i}}{2 \pi i}-z\right) \theta^{\prime}(0)}{\theta\left(\frac{x_{i}}{2 \pi i}\right) \theta(-z)} \prod_{\lambda, Q_{F_{\lambda}}} \frac{\theta\left(\frac{x_{\lambda, c}}{2 \pi i}\right) \theta\left(\frac{x_{\lambda, c}}{2 \pi i}+\lambda(g)-\lambda(h) \tau-z\right)}{\theta\left(\frac{x_{\lambda, c}}{2 \pi i}-z\right) \theta\left(\frac{x_{\lambda, c}}{2 \pi i}+\lambda(g)-\lambda(h) \tau\right)} e^{2 \pi i \lambda(h) z} \times \\
& \prod_{\varepsilon, Q_{N_{\varepsilon}}} \frac{\theta\left(\frac{x_{\varepsilon, a}}{2 \pi i}\right) \theta\left(\frac{x_{\varepsilon, a}}{2 \pi i}+\varepsilon(g)-\varepsilon(h) \tau-z\right)}{\theta\left(\frac{x_{\varepsilon}, a}{2 \pi i}-z\right) \theta\left(\frac{x_{\varepsilon, a}}{2 \pi i}+\varepsilon(g)-\varepsilon(h) \tau\right)} e^{2 \pi i \varepsilon(h) z} \times \\
& \prod_{j} \frac{\theta\left(\frac{\xi_{j}}{2 \pi i}\right) \theta\left(\frac{\xi_{j}}{2 \pi i}+\lambda_{j}(g)-\lambda_{j}(h) \tau-\left(-\alpha_{j}+1\right) z\right) \theta(-z)}{\theta\left(\frac{\xi_{j}}{2 \pi i}-z\right) \theta\left(\frac{\xi_{j}}{2 \pi i}+\lambda_{j}(g)-\lambda_{j}(h) \tau\right) \theta\left(-\left(-\alpha_{j}+1\right) z\right)} e^{-2 \pi i \alpha_{j} \lambda_{j}(h) z} \times \\
& \prod_{i} \frac{\theta\left(\frac{\Delta_{i}}{2 \pi i}-\left(-\alpha_{i}+1\right) z\right) \theta(-z)}{\theta\left(\frac{\Delta_{i}}{2 \pi i}-z\right) \theta\left(-\left(-\alpha_{i}+1\right) z\right)} .
\end{aligned}
$$

Here $x_{\lambda, c}, x_{\varepsilon, a}, \xi_{j}$, and $\Delta_{i}$ denote the equivariant Chern roots of the bundles, $Q_{F_{\lambda}}, Q_{N_{\varepsilon}}, Q_{\xi_{j}}$, and $Q_{\Delta_{i}}$, respectively. The above equality follows from lemma 7 .

Let $f: \widetilde{X}^{*} \rightarrow X^{*}$ be the blow-up along $Z$ with exceptional divisor $E$. Before proceeding further, it will be convenient to set up some new notation. Consider the collection $I=\left\{N_{\varepsilon}, F_{0}, F_{\lambda}, \xi_{j}, \Delta_{i}, E\right\}$. Let $\widetilde{Q}_{N_{\varepsilon}}=f^{*} Q_{N_{\varepsilon}}$. For $A \in I, A \neq N_{\varepsilon}, E$, let $\widetilde{Q}_{A}=f^{*} Q_{A} \otimes \mathcal{O}(-E)$. For ease of notation later on, we also define $\widetilde{Q}_{E}=\mathcal{O}(E)$. For $A \in I-\{E\}$, we let $\widetilde{V}_{A}$ denote the proper transform of $V_{A}$, and we let $\widetilde{V}_{E}$ simply equal $E$.

For $A \in I-\{E\}$ we define, for convenience of notation, $\lambda_{A} \in R((g, h))$ and $\alpha_{A} \in \mathbb{Q}$ as follows: For $A=N_{\varepsilon}, F_{\lambda}, \xi_{j}$, and $\Delta_{i}$, we let $\lambda_{A}=\varepsilon, \lambda, \lambda_{j}$, and $\lambda_{i}$, respectively. Otherwise, we set $\lambda_{A}=0$. Next, for $A=\xi_{j}$ or $\Delta_{i}$, we let $\alpha_{A}=\alpha_{j}$ or $\alpha_{i}$. Otherwise, we set $\alpha_{A}=0$.

Let $\widetilde{X^{*}}{ }_{\mu}^{g, h}$ be a connected component of $\widetilde{X^{*}}{ }^{g, h}$ which gets mapped to $X_{\gamma}^{g, h}$ under $f$. This space is the complete intersection of subvarieties $\widetilde{V}_{A}$ for $A$ in some indexing set $I_{\mu} \subset I$. For $A \in I_{\mu},\left.\widetilde{Q}_{A}\right|_{\widetilde{X}^{*}{ }_{\mu}, h}=N_{A, \mu} \otimes \widetilde{\lambda}_{A, \mu}$ for some $(g, h)$ character $\widetilde{\lambda}_{A, \mu}$ and some $(g, h)$-trivial bundle $N_{A, \mu}$. More precisely, we may canonically define $\widetilde{\lambda}_{E, \mu}=\lambda_{A^{\prime}}$, for some unique character $\lambda_{A^{\prime}}$ depending only on $\mu$, and then set $\widetilde{\lambda}_{A, \mu}=\lambda_{A}-\widetilde{\lambda}_{E, \mu}$. We extend the definition of $\widetilde{\lambda}_{A, \mu}$ by letting $\widetilde{\lambda}_{A, \mu}=0$ for $A \notin I_{\mu}$.

Finally, we define indices $\beta_{A}$ as follows: For $A=\xi_{j}, \Delta_{i}$, or $E$, we let $\beta_{A}$ be the coefficient of $\widetilde{V}_{A}$ as a divisor in $\widetilde{D}$. Otherwise, we let $\beta_{A}=0$.

Applying corollary 1 , lemma 9 , and the above definitions, we obtain the following convenient expression for $\mathcal{E} l l_{\text {orb }}\left({\widetilde{X^{*}}}_{\mu}{ }_{\mu}, h, \widetilde{D}\right)$ : 


$$
\begin{aligned}
& f^{*}\left\{\prod_{T X^{*}} \frac{\frac{x_{i}}{2 \pi i} \theta\left(\frac{x_{i}}{2 \pi i}-z\right) \theta^{\prime}(0)}{\theta\left(\frac{x_{i}}{2 \pi i}\right) \theta(-z)} \prod_{m, Q_{A}, A \in I} \frac{\theta\left(\frac{a_{m}}{2 \pi i}\right) \theta(-z)}{\frac{a_{m}}{2 \pi i} \theta\left(\frac{a_{m}}{2 \pi i}-z\right) \theta^{\prime}(0)}\right\} \times \\
& \\
& \prod_{\ell, \widetilde{Q}_{A}, A \in I} \frac{\frac{\tilde{a}_{\ell}}{2 \pi i} \theta\left(\frac{\tilde{a}_{\ell}}{2 \pi i}+\widetilde{\lambda}_{A, \mu}(g)-\widetilde{\lambda}_{A, \mu}(h) \tau-\left(-\beta_{A}+1\right) z\right) \theta^{\prime}(0)}{\theta\left(\frac{\tilde{a}_{\ell}}{2 \pi i}+\widetilde{\lambda}_{A, \mu}(g)-\widetilde{\lambda}_{A, \mu}(h) \tau\right) \theta\left(-\left(-\beta_{A}+1\right) z\right)} e^{2 \pi i\left(-\beta_{A}+1\right) \widetilde{\lambda}_{A, \mu} z} .
\end{aligned}
$$

Notice by our definition of $\tilde{\lambda}_{A, \mu}$ that $\sum\left(-\beta_{A}+1\right) \widetilde{\lambda}_{A, \mu}(h)=\sum\left(-\alpha_{A}+1\right) \lambda_{A}(h)$. By this observation and the projection formula, we are therefore reduced to proving the following formula:

$$
\begin{gathered}
f_{*} \sum_{\mu} \prod_{\ell, \widetilde{Q}_{A}, A \in I} \frac{\frac{\tilde{a}_{\ell}}{2 \pi i} \theta\left(\frac{\tilde{a}_{\ell}}{2 \pi i}+\widetilde{\lambda}_{A, \mu}(g)-\widetilde{\lambda}_{A, \mu}(h) \tau-\left(-\beta_{A}+1\right) z\right) \theta^{\prime}(0)}{\theta\left(\frac{\tilde{a}_{\ell}}{2 \pi i}+\widetilde{\lambda}_{A, \mu}(g)-\widetilde{\lambda}_{A, \mu}(h) \tau\right) \theta\left(-\left(-\beta_{A}+1\right) z\right)}= \\
\prod_{m, Q_{A}, A \in I-E} \frac{\frac{a_{m}}{2 \pi i} \theta\left(\frac{a_{m}}{2 \pi i}+\lambda_{A}(g)-\lambda_{A}(h) \tau-\left(-\alpha_{A}+1\right) z\right) \theta^{\prime}(0)}{\theta\left(\frac{a_{m}}{2 \pi i}+\lambda_{A}(g)-\lambda_{A}(h) \tau\right) \theta\left(-\left(-\alpha_{A}+1\right) z\right)} .
\end{gathered}
$$

Naturally, $\tilde{a}_{\ell}=\tilde{a}_{\ell}(A)$ denote the equivariant Chern roots of $\widetilde{Q}_{A}$ and $a_{m}=a_{m}(A)$ denote the equivariant Chern roots of $Q_{A}$.

Referring to the notation from section 9 for $A=N_{\varepsilon}, F_{0}, F_{\lambda}, \Delta_{k}, \xi_{j}$ and $i=$ $1, \ldots, \operatorname{rank}(A)$, let us define $x_{A, i} \in \mathbb{C}[\Sigma]=w_{\varepsilon, i}, x_{i}, y_{\lambda, i}, d_{k}, e_{j}$, respectively. For $A \in I$ and $i=1, \ldots, \operatorname{rank}(A)$ we define $\tilde{x}_{A, i} \in \mathbb{C}[\widetilde{\Sigma}]$ similarly. Define $F \in \mathbb{C}[|\Sigma|]$ to be the power series:

$$
F=\prod_{i, A \in I-E} \frac{\frac{x_{A, i}}{2 \pi i} \theta\left(\frac{x_{A, i}}{2 \pi i}+\lambda_{A}(g)-\lambda_{A}(h) \tau-\left(-\alpha_{A}+1\right) z\right) \theta^{\prime}(0)}{\theta\left(\frac{x_{A, i}}{2 \pi i}+\lambda_{A}(g)-\lambda_{A}(h) \tau\right) \theta\left(-\left(-\alpha_{A}+1\right) z\right)} .
$$

Define $F_{\mu} \in \mathbb{C}[|\widetilde{\Sigma}|]=$

$$
\prod_{i, A \in I} \frac{\frac{\tilde{x}_{A, i}}{2 \pi i} \theta\left(\frac{\tilde{x}_{A, i}}{2 \pi i}+\widetilde{\lambda}_{A, \mu}(g)-\widetilde{\lambda}_{A, \mu}(h) \tau-\left(-\beta_{A}+1\right) z\right) \theta^{\prime}(0)}{\theta\left(\frac{\tilde{x}_{A, i}}{2 \pi i}+\widetilde{\lambda}_{A, \mu}(g)-\widetilde{\lambda}_{A, \mu}(h) \tau\right) \theta\left(-\left(-\beta_{A}+1\right) z\right)} .
$$

Clearly $F$ is $\rho$ applied to expression 10 and $F_{\mu}$ is $\rho$ applied to the $\mu$-th summand in expression 11. By lemma 10, we are reduced to proving $\nu_{*} \sum_{\mu} F_{\mu}=F$. To do this, think of $\left(\Sigma, N_{\Sigma}\right)$ as the polyhedral complex associated to the toric variety $\mathbb{C}^{M}$ where $M=\operatorname{dim} \Sigma$. Let $\widetilde{\mathbb{C}^{M}}$ be the toric blow-up of $\mathbb{C}^{M}$ which corresponds to the polyhedral subdivision $\widetilde{\Sigma} \rightarrow \Sigma$ described in section 9. We may view $g$ and $h$ as elements of the big torus of $\mathbb{C}^{M}$, i.e., as elements of a finite index sup-lattice of $N_{\Sigma}$. Under this identification, $x_{A, i}(g)=\lambda_{A}(g)$.

The $(g, h)$-fixed components of $\widetilde{\mathbb{C}^{M}}$ are in one-one correspondence with the fixed components $\widetilde{X}^{*}{ }_{\mu}^{g, h}$ and in one-one correspondence with subcones $C_{\mu} \subset \widetilde{\Sigma}$. These are the cones of maximal dimension which correspond to affine open sets $U_{C_{\mu}}$ of the form $\mathbb{C}^{a} \times\left(\mathbb{C}^{*}\right)^{b}$, where the characters of the $(g, h)$-representation $\mathbb{C}^{a}$ are all non-trivial. For $C \supset C_{\mu}$, let $I(C)$ index the collection of piece-wise linear functions $\tilde{x}_{A, i}$ which are dual to $C$. Since $g$ and $h \in N_{\Sigma}$ lie inside $C_{\mu}$, it makes sense to evaluate $\left.\tilde{x}_{A, i}\right|_{C}$ at $g$ and $h$. One sees easily in fact that $\left.\tilde{x}_{A, i}\right|_{C}(g)=\tilde{\lambda}_{A, \mu}(g)$ and similarly for $\left.\tilde{x}_{A, i}\right|_{C}(h)$. When 
distinguishing between different cones, it will be convenient to denote the collection $\left\{\left.\tilde{x}_{A, i}\right|_{C}\right\}_{I(C)}$ by $\left\{\tilde{x}_{C, j}\right\}_{j=1}^{|I(C)|}$. With this notation, if $\tilde{x}_{C, j}=\left.\tilde{x}_{A, i}\right|_{C}$, we also define $\beta_{j}=\beta_{A}$. We define $x_{C, j}$ and $\alpha_{j}$ similarly when $C \subset \Sigma$.

From this it follows that for $C \supset C_{\mu}$ :

$$
\left.F_{\mu}\right|_{C}=\prod_{j=1}^{\operatorname{dim} C} \frac{\frac{\tilde{x}_{C, j}}{2 \pi i} \theta\left(\frac{\tilde{x}_{C, j}}{2 \pi i}+\tilde{x}_{C, j}(g)-\tilde{x}_{C, j}(h) \tau-\left(-\beta_{j}+1\right) z\right) \theta^{\prime}(0)}{\theta\left(\frac{\tilde{x}_{C, j}}{2 \pi i}+\tilde{x}_{C, j}(g)-\tilde{x}_{C, j}(h) \tau\right) \theta\left(-\left(-\beta_{j}+1\right) z\right)} .
$$

Otherwise, if $C_{\mu}$ is not contained in $C$, it is easy to see that $\left.F_{\mu}\right|_{C}=0$.

Now let $C_{\gamma} \subset \Sigma$ be the cone which corresponds to $\left(X^{*}\right)_{\gamma}^{g, h}$. Fix a cone $K \subset \Sigma$ containing $C_{\gamma}$. Let $\widetilde{\Sigma}_{K}$ denote the subdivision of $K$ inside $\widetilde{\Sigma}$. Each cone $C \subset \widetilde{\Sigma}_{K}$ with the same dimension as $K$ contains a unique cone $C_{\mu}$ for some $\mu$. Moreover, every cone $C_{\mu}$ is contained in one such cone $C \subset \widetilde{\Sigma}_{K}$. We therefore have that

$$
\left.\sum_{\mu} F_{\mu}\right|_{C}=\prod_{j=1}^{\operatorname{dim} K} \frac{\frac{\tilde{x}_{C, j}}{2 \pi i} \theta\left(\frac{\tilde{x}_{C, j}}{2 \pi i}+\tilde{x}_{C, j}(g)-\tilde{x}_{C, j}(h) \tau-\left(-\beta_{j}+1\right) z\right) \theta^{\prime}(0)}{\theta\left(\frac{\tilde{x}_{C, j}}{2 \pi i}+\tilde{x}_{C, j}(g)-\tilde{x}_{C, j}(h) \tau\right) \theta\left(-\left(-\beta_{j}+1\right) z\right)} .
$$

To complete the proof, it remains to show that $\left.\left(\nu_{*} \sum F_{\mu}\right)\right|_{K}=\left.F\right|_{K}$. By the push-forward formula for $\nu_{*}$, this is equivalent to proving the identity:

$$
\begin{aligned}
& \sum_{C \subset \widetilde{\Sigma}_{K}} \prod_{j=1}^{\operatorname{dim} K} \frac{\theta\left(\frac{\tilde{x}_{C, j}}{2 \pi i}+\tilde{x}_{C, j}(g)-\tilde{x}_{C, j}(h) \tau-\left(-\beta_{j}+1\right) z\right) \theta^{\prime}(0)}{\theta\left(\frac{\tilde{x}_{C, j}}{2 \pi i}+\tilde{x}_{C, j}(g)-\tilde{x}_{C, j}(h) \tau\right) \theta\left(-\left(-\beta_{j}+1\right) z\right)}= \\
& \prod_{j=1}^{\operatorname{dim} K} \frac{\theta\left(\frac{x_{K, j}}{2 \pi i}+x_{K, j}(g)-x_{K, j}(h) \tau-\left(-\alpha_{j}+1\right) z\right) \theta^{\prime}(0)}{\theta\left(\frac{x_{K, j}}{2 \pi i}+x_{K, j}(g)-x_{K, j}(h) \tau\right) \theta\left(-\left(-\alpha_{j}+1\right) z\right)} .
\end{aligned}
$$

Here the functions $\tilde{x}_{C, j}$ are regarded as linear combinations of the functions $x_{K, j}$. The above formula follows from theorem 7 of the preprint [18] or by lemma 8.1 of [7]. This completes the proof. $\square$

REMARK 7 . Note that setting $T=1$ in the above series of arguments gives us Borisov and Libgober's push-forward formula for the orbifold elliptic genus in [7]. It is interesting to compare their use of deformation to the normal cone to prove the non-equivariant push-forward formula to the use given here. In [7], deformation to the normal cone allows the authors to assume that normal bundle to the blow-up locus is the restriction of a globally defined holomorphic vectorbundle $Q$. In this situation, one has an explicit formula (see [11]) relating the Chern classes of $T X, T \widetilde{X}, Q$, and $\mathcal{O}(E)$. Using this formula, Borisov and Libgober are able to compare the pushforward of the blown-up orbifold elliptic genus to the original orbifold elliptic genus and show that they are the same by using a residue theorem argument. Here, our use of deformation to the normal cone is a bit stronger in the sense that we work entirely on the normal cone space $X^{*}$, making direct use of its topological data. On the normal cone space we are able to explicitly compute the push-forward of the orbifold elliptic genus by using an analogue of functorial localization, together with a useful theta-function identity.

Now, let $Z$ be a projective $\mathbb{Q}$-Gorenstein variety with log terminal singularities, and a regular $G \times T$ action. Let $f: X \rightarrow Z$ and $g: Y \rightarrow Z$ be two equivariant resolutions of singularities. We assume that the exceptional locus of both resolutions is a $G$-normal divisor with simple normal crossings. Define $D_{X}$ so that $K_{X}+D_{X}=$ 
$f^{*} K_{Z}$; define $D_{Y}$ similarly. Then the equivariant orbifold elliptic genera of $\left(X, D_{X}\right)$ and $\left(Y, D_{Y}\right)$ coincide. Indeed, by the equivariant version of the weak factorization theorem [1], we may connect $X$ to $Y$ by a sequence of equivariant blow-ups and blowdowns in such a way that the blow-ups at each intermediate pair $\left(X_{i}, D_{X_{i}}\right)$ occur along a smooth base with normal crossings with respect to the components of $D_{X_{i}}$. Moreover, the procedure described in [7] theorem 3.7 to make the intermediate pairs $\left(X_{i}, D_{X_{i}}\right) G$-normal extends to the $T$-equivariant case. Hence, by the equivariant change of variables formula, $E l l_{\text {orb }}\left(X, D_{X}, G\right)=\operatorname{Ell}_{\text {orb }}\left(Y, D_{Y}, G\right)$.

We will, however, require a slightly stronger version of the above result for the purposes of this paper:

Theorem 10. Let $f:\left(X, D_{X}\right) \rightarrow\left(Y, D_{Y}\right)$ be a $G \times T$-equivariant birational morphism between smooth, equivariant, $G$-normal log terminal pairs $\left(X, D_{X}\right)$ and $\left(Y, D_{Y}\right)$. Assume furthermore that $f^{*}\left(K_{Y}+D_{Y}\right)=K_{X}+D_{X}$. Then $f_{*} \mathcal{E} l l_{\text {orb }}\left(X, D_{X}, G\right)=$ $\mathcal{E} l l_{\text {orb }}\left(Y, D_{Y}, G\right)$.

Proof. The weak factorization theorem allows us to factor $f$ into a sequence of equivariant blow-ups and blow-downs

$$
X=X_{0} \rightarrow X_{1} \rightarrow \cdots \cdots X_{k}=Y
$$

such that for some intermediate index $i_{0}$ the maps $X_{i} \rightarrow X$ are morphisms for $i \leq i_{0}$ and the maps $X_{i} \rightarrow Y$ are morphisms for $i \geq i_{0}$. Moreover, by [7] theorem 3.7, we may still guarantee that all the intermediate varieties are $G$-normal with respect to the appropriate divisors. Note that since $f$ is itself a smooth morphism, we may conclude that the maps $X_{i} \rightarrow Y$ are smooth morphisms for all $i$.

We now apply induction on $k$. For $k=1$, the theorem is obvious. Otherwise, consider the intermediate variety $X_{1}$. By assumption, $X_{1} \neq X, Y$. Either $X \rightarrow X_{1}$ is a blow-up or blow-down. Suppose first that $X \leftarrow X_{1}$ is a blow-down. Call this morphism $g$. Define $D_{1}$ so that $K_{X_{1}}+D_{1}=g^{*}\left(K_{X}+D_{X}\right)$. Note that if $h: X_{1} \rightarrow Y$ is the morphism $f \circ g$, then $K_{X_{1}}+D_{1}=h^{*}\left(K_{Y}+D_{Y}\right)$. By the change of variables formula, $\mathcal{E} l l_{\text {orb }}\left(X, D_{X}, G\right)=g_{*} \mathcal{E} l l_{\text {orb }}\left(X_{1}, D_{1}, G\right)$. Therefore $f_{*} \mathcal{E} l l_{\text {orb }}\left(X, D_{X}, G\right)=$ $h_{*} \mathcal{E} l l_{\text {orb }}\left(X_{1}, D_{1}, G\right)$. But this is equal to $\mathcal{E} l l_{\text {orb }}\left(Y, D_{Y}, G\right)$ by the induction hypothesis.

The case in which $X \rightarrow X_{1}$ is a blow-down is proved similarly.

11. The Equivariant McKay Correspondence. Here we use the results from the preceding sections to prove an equivariant analogue of the McKay Correspondence for the elliptic genus. As a corollary, we will arrive at an equivariant version of the DMVV formula.

Let $X$ be a smooth projective variety with a $G \times T$ action, where $G$ is a finite group and $T$ is a compact torus. Let $\psi: X \rightarrow X / G$ be the quotient morphism. Assume that $X / G$ has an equivariant crepant resolution $V$ and that $\psi^{*}\left(K_{X / G}\right)=K_{X}$. Let $F \subset X$ be a fixed component of the $G \times T$-action, and let $\{P\} \subset V$ denote the fixed components in $V$ which get mapped to $\psi(F)$. Then:

THEOREM 11.

$$
\int_{F} \frac{\mathcal{E} l l_{\text {orb }}(X, G)}{e(F)}=\sum_{P} \int_{P} \frac{\mathcal{E} l l(V)}{e(P)} .
$$

Proof. Let $Z \rightarrow V$ be a sequence of equivariant blow-ups of $V$ so that the exceptional locus of the resolution $\pi: Z \rightarrow X / G$ is a divisor with simple normal 
crossings. Let $D_{Z}$ be the divisor on $Z$ such that $K_{Z}+D_{Z}=\pi^{*} K_{X / G}$. Define $\hat{Z}_{0}$ to be the normalization of $Z$ in the function field of $X$. By Abhyankar's Lemma, the induced map $\mu_{0}: \hat{Z}_{0} \rightarrow Z$ is a toroidal morphism of toroidal embeddings. Let $\hat{Z}$ be a projective toroidal resolution of singularities of $\hat{Z}_{0}$, and define $D_{\hat{Z}}$ so that $K_{\hat{Z}}+D_{\hat{Z}}=\mu^{*}\left(K_{Z}+D_{Z}\right)$, where $\mu: \hat{Z} \rightarrow Z$ is the obvious map. We may further assume that $\hat{Z}$ has a $G$ action, and that the pair $\left(\hat{Z}, D_{\hat{Z}}\right)$ is $G$-normal. (see [2]). We obtain the following commutative diagram:

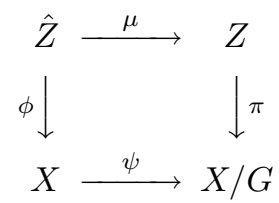

Here, the vertical arrows are resolutions of singularities, and the horizontal arrows are birational to a quotient by $G$. It is clear that all the constructions involved (normalization, blow-up along a $T$-invariant ideal sheaf) are $T$-equivariant, and that consequently the above morphisms are $T$-equivariant. Since $\phi^{*}\left(K_{X}\right)=K_{\hat{Z}}+D_{\hat{Z}}$, we have that $\mathcal{E} l l_{\text {orb }}^{T}(X, G)=\phi_{*} \mathcal{E} l l_{\text {orb }}\left(\hat{Z}, D_{\hat{Z}}, G\right)$. Let $\{L\} \subset \hat{Z}$ denote the fixed components of $\hat{Z}$ which map to $F$. By functorial localization:

$$
\int_{F} \frac{\mathcal{E} l l_{\text {orb }}(X, G)}{e(F)}=\sum_{L} \int_{L} \frac{\mathcal{E} l l_{\text {orb }}\left(\hat{Z}, D_{\hat{Z}}, G\right)}{e(L)} .
$$

Now, by theorem $7, \mu_{*} \mathcal{E} l l_{\text {orb }}\left(\hat{Z}, D_{\hat{Z}}, G\right)=\mathcal{E} l l\left(Z, D_{Z}\right)$. Let $\{K\} \subset Z$ denote the fixed components which get mapped to $\psi(F)$ under the resolution $Z \rightarrow X / G$. Clearly $\{L\}=$ $\phi^{-1} F=\phi^{-1} \psi^{-1} \psi(F)=\mu^{-1} \pi^{-1} \psi(F)=\mu^{-1}\{K\}$. Thus, by functorial localization applied to $\mu_{*}$, we have:

$$
\sum_{L} \int_{L} \frac{\mathcal{E} l l_{\text {orb }}\left(\hat{Z}, D_{\hat{Z}}, G\right)}{e(L)}=\sum_{K} \int_{K} \frac{\mathcal{E} l l\left(Z, D_{Z}\right)}{e(K)} .
$$

Finally, since $\{K\}$ denotes the fixed components of $Z$ which get mapped to $\{P\}$, functorial localization applied to $Z \rightarrow V$ completes the proof. $\square$

REMARK 8. In case $X / G$ possesses no crepant resolution, the above proof shows that $\int_{F} \frac{\mathcal{E} l l_{\text {orb }}(X, G)}{e(F)}=\sum_{P} \int_{P} \frac{\mathcal{E} l l\left(Z, D_{Z}\right)}{e(P)}$, where in this formula $\{P\}$ denotes the fixed components of $Z$ which get mapped to $\psi(F)$.

We now discuss some corollaries of the above result. We begin with a proof of the equivariant DMVV conjecture. Let $\mathbb{P}_{2}^{(n)}=\left(\mathbb{P}_{2}\right)^{n} / S_{n}$ denote the $n$th symmetric product of the projective plane. The natural group action of $T=S^{1} \times S^{1}$ on $\mathbb{P}_{2}$ extends to $\left(\mathbb{P}_{2}\right)^{n}$ in the obvious manner, and commutes with the action of $S_{n}$. The action of $T$ on $\mathbb{P}_{2}$ also extends to $\mathbb{P}_{2}^{[n]}$, the Hilbert scheme of $n$ points on $\mathbb{P}_{2}$, and the Hilbert-Chow morphism $\mathbb{P}_{2}^{[n]} \rightarrow \mathbb{P}_{2}^{(n)}$ is an equivariant crepant resolution.

Sitting inside $\mathbb{P}_{2}^{(n)}$ is the open variety $\left(\mathbb{C}^{2}\right)^{(n)}$. It has a single $T$-fixed point $p$, which is the image under the quotient morphism of the $S_{n} \times T$ fixed point $(0,0) \times \ldots \times(0,0) \in$ $\left(\mathbb{C}^{2}\right)^{n}$. The pre-image of $\left(\mathbb{C}^{2}\right)^{(n)}$ under the Hilbert-Chow morphism is just $\left(\mathbb{C}^{2}\right)^{[n]}$. Hence, the pre-image of $p$ under the Hilbert-Chow morphism is simply the collection 
of $T$-fixed points of $\left(\mathbb{C}^{2}\right)^{[n]}$. If $\left(u_{1}, u_{2}\right)$ denote the equivariant parameters of the $T$ action, let $t_{j}=e^{2 \pi i u_{j}}$. Then the above theorem implies that:

$$
\operatorname{Ell}_{\text {orb }}\left(\left(\mathbb{C}^{2}\right)^{n}, S_{n}, t_{1}, t_{2}\right)=\operatorname{Ell}\left(\left(\mathbb{C}^{2}\right)^{[n]}, t_{1}, t_{2}\right) .
$$

Note that the LHS involves equivariant data associated to the single fixed point $p$, whereas the RHS involves a sum of equivariant data associated to partitions of $n$.

For $z$ the complex parameter appearing in the definition of the elliptic class and $\tau$ the lattice parameter used in the definition of the Jacobi theta function, let $y=e^{2 \pi i z}$ and $q=e^{2 \pi i \tau}$. Viewing the equivariant elliptic indices as formal power series in the variables $q, y, t_{1}$, and $t_{2}$, and applying theorem 3.1 of [15], we have the following equivariant analogue of the DMVV formula:

THEOREM 12. Expand

$$
\operatorname{Ell}\left(\mathbb{C}^{2}, t_{1}, t_{2}\right)=y^{-1} \prod_{i=1}^{2} \frac{1-y t_{i}^{-1}}{1-t_{i}^{-1}} \frac{\prod_{n=1}^{\infty}\left(1-y t_{i}^{-1} q^{n}\right)\left(1-y^{-1} t_{i} q^{n}\right)}{\prod_{n=1}^{\infty}\left(1-t_{i}^{-1} q^{n}\right)\left(1-t_{i} q^{n}\right)}
$$

as $\sum_{m \geq 0, \ell, k} c(m, \ell, k) q^{m} y^{\ell} t_{1}^{k_{1}} t_{2}^{k_{2}}$. Then

$$
\sum_{n>0} p^{n} \operatorname{Ell}\left(\left(\mathbb{C}^{2}\right)^{[n]}, t_{1}, t_{2}\right)=\prod_{m \geq 0, n>0, \ell, k} \frac{1}{\left(1-p^{n} q^{m} y^{\ell} t_{1}^{k_{1}} t_{2}^{k_{2}}\right)^{c(n m, \ell, k)}}
$$

We next discuss the equivariant elliptic genus analogue of the classical McKay correspondence, which was originally proved in [18]. Let $G \subset S U(2)$ be a finite subgroup. $G$ acts on $\mathbb{C}^{2}$ in the obvious fashion, and commutes with the diagonal action of $T=S^{1}$. Let $V \rightarrow \mathbb{C}^{2} / G$ be the crepant resolution of singularities. The action of $T$ lifts to $V$, and the fixed components of this action are compact.

TheOREM 13. $\operatorname{Ell}_{\text {orb }}\left(\mathbb{C}^{2}, G, t\right)=\operatorname{Ell}(V, t)$.

Proof. View $\mathbb{C}^{2}$ as an open subset of $\mathbb{P}^{2}$, with the action of $G$ and $T$ extending to $\mathbb{P}^{2}$ in the obvious manner. The space $\mathbb{P}^{2} / G$ still has only an isolated singularity at the image of the origin $[0: 0: 1]$. Hence $\mathbb{P}^{2} / G$ has an equivariant crepant resolution which is a compactification of $V$. The above theorem then follows by letting $F=(0,0)$ in theorem 11 .

\section{Appendix.}

Lemma 11. Let $f: X \rightarrow Y$ be a T-map of smooth compact simply connected complex manifolds. Let $D \subset Y$ be a $T$-invariant divisor and let $E_{i}$ be $T$-invariant normal crossing divisors on $X$ such that $f^{*} D=\sum a_{i} E_{i}$ as Cartier divisors. Then for any $\varepsilon$-regular neighborhood $U_{\varepsilon}$ of $D$ there exist generators $\Theta_{E_{i}}^{T}$ for $c_{1}^{T}\left(E_{i}\right)$ and $\Theta_{D}^{T}$ for $c_{1}^{T}(D)$ with the following properties:

(1) $\Theta_{D}^{T}$ has compact support in $U_{\varepsilon}$ and $\Theta_{E_{i}}^{T}$ have compact support in $f^{-1}\left(U_{\varepsilon}\right)$.

(2) $f^{*} \Theta_{D}^{T}=\sum a_{i} \Theta_{E_{i}}^{T}+d_{T}(\eta)$ on the level of forms, where $\eta$ is a $T$-invariant form with compact support in $U_{\varepsilon}$.

(3) $\Theta_{D}^{T}$ and $\Theta_{E_{i}}^{T}$ represent the extension by zero of the equivariant Thom classes of the varieties $D$ and $E_{i}$

The only real issue above is to ensure that $\eta$ has compact support in the desired neighborhood. 
Proof. We first solve this problem in the non-equivariant category. For $V$ any Cartier divisor, denote by $L_{V}$ the line bundle it induces. Let $U_{\varepsilon}$ be a $T$-invariant tubular neighborhood of $D$ of radius $\varepsilon$. Outside $U_{\frac{\varepsilon}{2}}$, the constant function 1 is a section of $L_{D}$. Define a metric $h_{f a r}$ in this region by $h_{f a r}=\|1\|^{2} \equiv 1$. Let $h_{\text {near }}$ be a metric inside $U_{\varepsilon}$. Piece the two metrics into a global metric $h$ on $L_{D}$ using a partition of unity. The first chern class of $L_{D}$ is then represented by the form $\Theta_{D}=\frac{i}{2 \pi} \bar{\partial} \partial \log h$. This form clearly has compact support in $U_{\varepsilon}$.

Let $U_{\varepsilon_{i}}$ be tubular neighborhoods of $E_{i}$. Choose $\varepsilon_{i}$ small enough so that each of these neighborhoods is contained in $f^{-1} U_{\varepsilon}$. Define metrics $h_{i}$ on $E_{i}$ in a manner analogous to the above construction of $h$. Clearly the forms $\Theta_{E_{i}}=\frac{i}{2 \pi} \bar{\partial} \partial \log h_{i}$ have compact support in $U_{\varepsilon_{i}}$ and represent the first chern classes of $L_{E_{i}}$.

We have two natural choices for a metric on $f^{*} L_{D}$, namely $f^{*} h$ and $h_{1}^{a_{1}} \cdots h_{k}^{a_{k}}$. Choose a smooth nonzero function $\varphi$ so that $f^{*} h=\varphi h_{1}^{a_{1}} \cdots h_{k}^{a_{k}}$. Notice that $\varphi \equiv 1$ outside $f^{-1} U_{\varepsilon}$. We have:

$$
\bar{\partial} \partial \log f^{*} h=\bar{\partial} \partial \log \varphi+\sum_{i} a_{i} \bar{\partial} \partial \log h_{i}
$$

But this implies that $f^{*} \Theta_{D}=\sum_{i} a_{i} \Theta_{E_{i}}+\frac{i}{2 \pi} \bar{\partial} \partial \log \varphi$. If we let $d^{c}=\frac{i}{4 \pi}(\bar{\partial}-\partial)$, we may write this last equation as:

$$
f^{*} \Theta_{D}=\sum_{i} a_{i} \Theta_{E_{i}}-d d^{c} \log \varphi
$$

The form $\eta=-d^{c} \log \varphi$ clearly has compact support in $f^{-1} U_{\varepsilon}$. It remains to argue that $\Theta_{D}$ and $\Theta_{E_{i}}$ represent the Thom classes of $D$ and $E_{i}$. It is a standard fact that these classes are Poincaré duals to their respective divisors. If a divisor is homologously non-trivial, then clearly its Thom classes coincides with its Poincaré dual. If a divisor is homologously trivial, then it must follow that the extension by zero of its Thom class is trivial. Either way this implies that the above classes represent the extension by zero of the Thom classes of their respective divisors. This completes the non-equivariant portion of the proof.

By averaging over the group $T$, we may assume that all the forms above are $T$-invariant. For notational simplicity, let us assume that $T=S^{1}$. Let $V$ be the vectorfield on $X$ induced by the $T$-action. Let $g_{i}$ be the functions compactly supported in $f^{-1} U_{\varepsilon}$ which satisfy the moment map equation $i_{V} \Theta_{E_{i}}=d g_{i}$. Similarly, let $W$ be the vectorfield on $Y$ defined by the $T$-action and define $g$ so that it satisfies $i_{V} \Theta_{D}=d g$ and has support inside $U_{\varepsilon}$. Note that since $f$ is $T$-equivariant, $i_{V} f^{*} \Theta_{D}=f^{*} i_{W} \Theta_{D}=$ $f^{*} d g$. We then have $d(g \circ f)=\sum_{i} a_{i} d g_{i}+i_{V} d \eta=\sum_{i} a_{i} d g_{i}-d i_{V} \eta$. Hence $g \circ f=$ $\sum_{i} a_{i} g_{i}-i_{V} \eta$. But this implies that:

$$
f^{*}\left(\Theta_{D}+g\right)=\sum_{i} a_{i}\left(\Theta_{E_{i}}+g_{i}\right)+\left(d-i_{V}\right) \eta
$$

But this is precisely the relation we wish to in the equivariant cohomology.

Lemma 12. (Excess Intersection Formula) Let $X$ be a smooth compact variety with irreducible normal crossing divisors $D_{1}, \ldots, D_{k}$. For $I \subset\{1, \ldots, k\}$ denote by $X_{I, j}$ the $j$ th connected component of $\cap_{I} D_{i}$ and by $\Phi_{I, j}$ its Thom class. Fix irreducible subvarieties $X_{I_{1}, j_{1}}$ and $X_{I_{2}, j_{2}}$. For $I_{0}=I_{1} \cup I_{2}$, let $X_{I_{0}, j}$ be the irreducible components of $X_{I_{1}, j_{1}} \cap X_{I_{2}, j_{2}}$. Then: 


$$
\Phi_{I_{1}, j_{1}} \wedge \Phi_{I_{2}, j_{2}}=\sum_{I_{0}, j} \Phi_{I_{0}, j} \prod_{I_{1} \cap I_{2}} \Phi_{i}
$$

Proof. Let $N_{I, j}$ be tubular neighborhoods of $X_{I, j}$ which are disjoint for each indexing set $I$ and which satisfy $N_{I, j} \subset N_{I^{\prime}, j^{\prime}}$ for $X_{I, j} \subset X_{I^{\prime}, j^{\prime}}$. If we choose $\Phi_{i}$ to have compact support in a sufficiently small tubular neighborhood of $D_{i}$, then $\prod_{I} \Phi_{i}$ will have compact support in $\coprod_{j} N_{I, j}$. Moreover, the extension by zero of $\left.\left(\prod_{I} \Phi_{i}\right)\right|_{N_{I, j}}$ will represent the Thom class of $X_{I, j}$. We may also ensure that $\Phi_{I_{1}, j_{1}} \wedge \Phi_{I_{2}, j_{2}}$ has compact support in $\coprod_{j} N_{I_{0}, j}$. Thus:

$$
\begin{gathered}
\Phi_{I_{1}, j_{1}} \wedge \Phi_{I_{2}, j_{2}}=\left.\sum_{I_{0}, j}\left(\prod_{I_{1}} \Phi_{i} \prod_{I_{2}} \Phi_{i}\right)\right|_{N_{I_{0}, j}}=\left.\sum_{I_{0}, j}\left(\prod_{I_{0}} \Phi_{i} \prod_{I_{1} \cap I_{2}} \Phi_{i}\right)\right|_{N_{I_{0}, j}}= \\
\left.\sum_{I_{0}, j}\left(\prod_{I_{0}} \Phi_{i}\right)\right|_{N_{I_{0}, j}} \prod_{I_{1} \cap I_{2}} \Phi_{i} .
\end{gathered}
$$

This yields the desired formula.

REMARK 9. Note that the above proof clearly extends to the equivariant category.

\section{REFERENCES}

[1] D. Abramovich, K. Karu, K. Matsuki, J. Wlodarczyk, Torification and factorization of birational maps, J. Amer. Math. Soc., 15 (2002), pp. 531-572.

[2] D. Abramovich, J. WAng, Equivariant resolution of singularities in characteristic 0Math. Res. Lett., 4 (1997), pp. 427-433.

[3] M.F. АтіYAн, R. Вотт, The moment map and equivariant cohomology, Topology, 23 (1984), pp. $1-28$.

[4] M. Atiyah, I. Singer, The index of elliptic operators III, Ann. of Math., (2) 87 (1968), pp. 546604.

[5] V.V. Batyrev, Non-Archimedian integrals and stringy Euler numbers of log-terminal pairs, J. Eur. Math. Soc. (JEMS), 1 (1999), pp. 5-33.

[6] L. Borisov, A. Libgober, Elliptic genera of singular varieties, Duke Math. J., 116 (2003), pp. 319-351.

[7] L. Borisov, A. Libgober, McKay correspondence for elliptic genera, Ann. of Math, 161 (2005), pp. 1521-1569.

[8] M. Brion, M. Vergne, An equivariant Riemann-Roch theorem for complete, simplicial toric varieties, J. Reine Angew. Math., 482 (1997), pp. 67-92.

[9] R. Dijkgraaf, G. Moore, E. Verlinde, H. Verlinde, Elliptic genera of symmetric products and second quantized strings, Comm. Math. Phys., 185 (1997), pp. 197-209.

[10] W. Fulton, Intersection theory, second edition, Springer Verlag, Berlin 1998.

[11] W. Fulton, Introduction to toric varieties, Ann. of Math Studies 131, Princeton University Press, Princeton, N.J., 1993.

[12] F. Hirzebruch, T. Berger, R. Jung, Manifolds and modular forms, with appendices by NilsPeter Skoruppa and Paul Baum, Aspects of Mathematics, E20. Friedr. Vieweg \& Sohn, Braunschweig, 1992.

[13] B. Lian, K. Liu, S.T. YAu, Mirror principle I, Surveys in differential geometry: differential geometry inspired by string theory, 5, pp. 405-454, Int. Press, Boston, MA, 1999.

[14] B. Lian, K. LiU, S.T. YAU, Mirror principle II, Surveys in differential geometry: differential geometry inspired by string theory, 5, pp. 455-509, Int. Press, Boston, MA, 1999.

[15] J. Li, K. LiU, J. Zhou, Topological string partition functions as equivariant indices, Asian J. Math., 10 (2006), pp. 81-114.

[16] K. Liu, On elliptic genera and theta-functions, Topology, 35 (1996), pp. 617-640.

[17] C.-L. WANG, K-equivalence in birational geometry and characterizations of complex elliptic genera, J. Algebraic Geom., 12 (2003), pp. 285-306.

[18] R. WAELDER, Equivariant elliptic genera, preprint, math.AG/0603521.

[19] E. Witten, Supersymmetry and Morse theory, J. Differential Geom., 17 (1982), pp. 661-692. 